it has been reproduced from the best available copy to permit the troadest possible availability.

\section{LA-10045}

UC-15

Issued: August 1984

LA- -10045

DE85 002347

\title{
The Use of Calibration Standards and the Correction for Sample Self-Attenuation in Gamma-Ray Nondestructive Assay
}

\author{
J. L. Parker
}

\section{DISCLAIMER}

erepared as an account of work sponsored by an agency of the United States This repott weither the United States Government nor any agency thereor, nor any of their Government. Neither the Unanty, express or implied, ar assumes any legal liabihty or respons employees, makes any warcy, completeness, or usefulness of any information, apparatus, prodsch, Referbility for the accuracy, completeness, or use would not infringe privately owed res trademark,

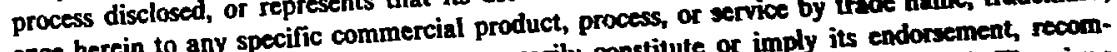
ence herein to any specife does not necessarily constitute or imply its endereof. The views manujacturer, or otherwise does not necessation, or favoring by the United States Government or any agency thereor. The of the

and opinions of authors expressed herein do noted 


\section{CONTENTS}

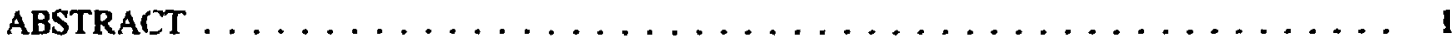

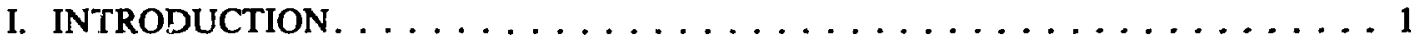

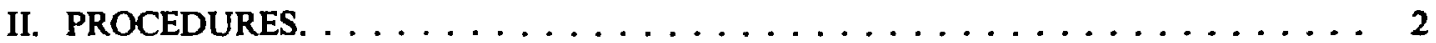

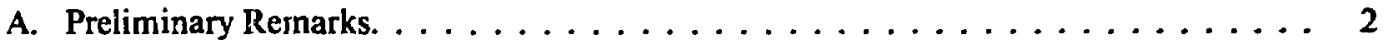

B. General Description of Assay Procedure . . . . . . . . . . . . . . . 3

C. Brief Discussion of Data Acquisition Rate, Deadtime, and Pileup . . . . . . . . . 4

1. Determination of the Raw Data Acquisition Rate . . . . . . . . . . . . . . 4

2. The Correction for Deadtime and Pileup. . . . . . . . . . . . . . . . 4

D. General Discussion of CF(AT) .................... 5

1. Necessary Assumptions for Determination of CF(AT) . . . . . . . . . . . 5

2. Methods for Determining the Sample Linear Attenuation Coefficient . . . . . . . . 8

III. FORMAL DEFINITION OF CORRECTION FOR SELF-ATTENUATION . . . . . . 9

A. The General Definition $\ldots \ldots \ldots \ldots \ldots \ldots \ldots \ldots \ldots \ldots$

B. Useful Sperified Shapes . . . . . . . . . . . . . . . . . . 10

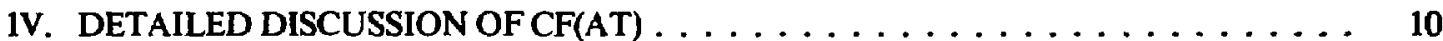

A. Important Parameters and General Trends . . . . . . . . . . . . . . . 10

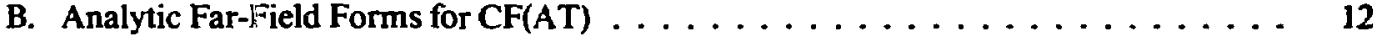

1. Box-Shaped Samples . . . . . . . . . . . . . . . . . . . . 12

2. Cylindrically Shaped Samples. . . . . . . . . . . . . . . . . 13

3. Spherically Shaped Samples . . . . . . . . . . . . . . . . . . . . . 14

C. Numeric Computation in the Near Field. . . . . . . . . . . . . . . . 15

1. General Discussion. . . . . . . . . . . . . . . . . . . 15

2. A Useful One-Dimensional Model . . . . . . . . . . . . . . . . . 15

3. A Useful Two-Dimensional Model . . . . . . . . . . . . . . . . . . . . 18

4. An Example of a Three-Dimensional Model. . . . . . . . . . . . . . . 21

5. Approximate Forms and Interpolation. . . . . . . . . . . . . 26

6. The Effects of Absolute and Relative Error in CF(AT) . . . . . . . . . . 28

7. Considerations of the Precision of CF(AT) and of TCR . . . . . . . . . 30

V. REVIEW OF FACTORS YOOVERNING THE NUMBER OF STANDARDS USED . . 31

REFERENCES ........................... 32 


\title{
THE USE OF CALIBRATION STANDARDS AND \\ TIE CORRECTION FOR SAMPLE SELF-ATTENUATION IN \\ GAMMA-RAY NONDESTRUCTIVE ASSAY
}

by

\author{
J. L. Parker
}

\begin{abstract}
The efficient use of appropriate calibration standards and the correction for the attenuation of the gamma rays within an assay sample by the sample itself are two important and closely related subjects in gamma-ray nondestructive assay. Much research relating to those subjects has been done in the Nuclear Safeguards Research and Development program at the Los Alamos National Laboratory since 1970. This report brings together most of the significant results of that research. Also discussed are the nature of appropriate calibration standards and the necessary conditions on the composition, size, and shape of the samples to allow accurate assays. Procedares for determining the correction for the sample self-attenuation are described at length including both general principles and several specific usefil cases. The most wsefil concept is that knowing the linear attenution coeficient of the sample (which can usually be determined) and the size and shape of the sample and its position relative to the detector permits the computation of the correction factor for the self-attenenation. A major objective of the report is to explain how the procedures for determining the selfattenuation correction factor can be applied so that calibration standards can be entirely appropriate without being particalarly similar, either physically or chemically, to the items to be assayed. This permits minimization of the number of standards required to assay items with a wide range of size, shape, and chemical composition.
\end{abstract}

\section{INTRODUCTION}

The proper use of calibration standards and the correction for the attenuation of the gamma rays emitted within an assay sample by the sample itself are two important and closely related subjects in gamma-ray nondestructive assay (NDA). In principle, calibration standards are not needed if the detector efficiency is accurately known as a function of source position and energy, if the counting geometry and the sample size and shape are accurately known, and if the gamma-ray emission rates are accurately known for the isotope(s) being assayed. However, it is tedious to characterize a detector efficiency with sufficient accuracy, and there are still significant uncertainties in the values of the specific activities for many gamma rays from many isotopes. Use of calibration standards reduces or eliminates the need to exactly characterize the detector efficiency, to know the counting peometry accurately, or to know well the specific activities of the isotopes of interest.* Thus, the calibration of a gamma-ray NDA system is more quickly and accurately determined with appropriate standards than is now possibie without them, making the use of calibration standards highly desirable when appropriate ones are available.

However, whether or not calibration standards are used, sample self-attenuation corrections must be made

*Rather rough values for efficiencies and emission rates are usually readily available and serve a useful purpose in estimating counting rates and assay precisions when setting up an appropriate assay seometry. However, they are not usually good enough to create a calibration that will produce accurate assays. 
for both the calibration standards and the unknown samples to be assayed.

In the early days of gamma-ray assay for safeguards purposes (roughly the late 1960 s), the problems of detector response per unit activity and of sample selfattenuation were often addressed by the use of "representative" standards, which were both chemically and physically similar to the samples being assayed. Such a procedure was probably wise considering the rudimentary state of NDA equipment and the general lack of careful anaiysis of the many assay problems. However, both equipment and the analysis of the problems have improved greatly so that, for gamma-ray assay at least, the requirement for physical and chemical similarity between standards and unknowns can be greatly relaxed. Unfortunately, the most authoritative currently available guide for calibrating NDA systems (ANSI N15.20-1975) ${ }^{1}$ was written about 1974 , before it was realized how nearly unnecessary the representative standard is for gamma-ray assay. As a result, that document rather firmly insists that for all NDA methods a calibration standard is "an item physically and chemically similar to the items to be assayed," a restriction no longer necessary for gamma-ray assay.

ANSI N15.20-1975 also insists that calibration standards "must be chosen so that their contained masses of the nuclide(s) of interest span the mass range expected for the items to be assayed." However, this restriction too may now be considerably relaxed, again because of advancements in both equipment and the physical analysis of assay problems.

If both these restrictions were strictly interpreted, many standards would be needed because of the many classes of materials for which NDA measurements are wanted and the numerous types of containers used for those materials. The objective of this report is to explain procedures whereby calibration standards for gammaray assay can be entirely appropriate without being particularly similar, either physically or chemically, to the items to be assayed. The obvious advantage is that relatively few standards can usually be used to calibrate gamma-ray assay systems for the accurate assay of items covering a wide range of size, shape, chemical composition, and mass of the nuclide(s) to be assayed with large consequent savings of time, material, effort, and expense. The procedures, though recognized and applied in several facilities, will be described primarily as they have developed in the Nuclear Safeguards Research and Development program at the Los Alamos National Laboratory. Several staff members have contributed significantly to that development. The procedures to be described and their applications are documented as they developed in many reports and papers. An effort will be made to give a sufficiently comprchensive treatment that it alone will be useful to NDA practitioners.

Most of the examples considered concern the assay of isotopes of uranium and plutonium in various chemical forms and sample shapes. There are two reasons for that emphasis. First, the Nuclear Safeguards Research and Development program is primarily concerned with NDA of those elements. Second, because of the relatively high gamma-ray mass attenuation coefficients of the high-Z elements, they represent the most difficult problems for gamma-ray NDA methods. The procedures to be described are equally applicable to the NDA of any gamma-ray-emitting isotopes.

\section{PROCEDURES}

\section{A. Preliminary Remarks}

The procedures and methods described herein are most cleanly and usefully applied with high-resolution gamma-ray detectors. Germanium detectors are most frequently employed for high-accuracy assays using photon energies greater than about $50 \mathrm{keV}$, and silicon detectors are most frequently used for applications employing photons of energies less than about $50 \mathrm{keV}$. The methods and correction factors may be used for assays with low-resolution detectors such as NaI(TI) scintillators, but additional care must be exercised to avoid unnecessary error, and the ultimate accuracy will be not be as good. The reason for the preference for highresolution detectors lies partially in the effects of smallangle Compton scattering, partially in the difficulties of background subtraction, and partially in the complications caused by overlapping peaks in complex spectra. With $\mathrm{NaI}(\mathrm{Tl})$ detectors, for example, whose full-energy peaks are 10-20 times as wide for a given energy as those of germanium detectors, a significant fraction of emitted photons can suffer a small-angle Compton scattering, lose a few kilovolts of energy, and still be counted in the full-energy peak. This condition makes it difficult to compute the fraction of photons emitted by a sumple that will undergo interactions, which will give rulses that can be stored in the full-energy peaks. It is also more difficult with low-resolution detectors to estimate the magnitude of the Compton background continuum, which is subtracted from under the full-energy peaks. Finally, it is m uch easier to extract the information from a spectrum when the full-energy peaks (which carry the necessary information) do not overlap, and clearly the possibility of overlap is much less for a high-resolution detector. All three of these problems are 10-20 times less 
severe in germanium detectors than in $\mathrm{NaI}(\mathrm{Tl})$ detectors, hence the general preference for germanium or other high-resolution detectors.

Of course, there are situations in which low-resolution detectors will be and sometimes must be chosen. Both germanium and silicon high-resolution detectors are generally more expensive and less efficient than the lower resolution scintillators. When the best accuracy is not required and a low-resolution detector can get adequate results, budgetary common sense suggests their use. Often the necessity for high sensitivity, even at the expense of accuracy, demands the use of a high-efficiency but low-resolution detector. High-resolution germanium and silicon detectors must, in general, be cooled when used and are thus not as easily used in portable assay systems as are the more compact but lower resolution detectors. Hence, the low-resolution units will often be used for the sake of portability, even at the sacrifice of some accuracy.

However, in spite of all these valid reasons, and more, for the use of low-resolution detectors when appropriate, it is still true that the most accurate (least biased) gamma-ray assays will be obtained with highresolution detectors when it is possible to use them.

The most unpleasant and important fact in applying gamma-ray spectroscopy to NDA is that the raw rate at which data are collected for a given gamma ray emitted by the sample is not usually proportional to the amount of nuclide emitting the gamma ray. Two reasons for this lack of proportionality are the rate-dependent electronic processes of deadtime and pulse pileup and the selfattenuation of the sample. Accurate gamma-ray assays demand accurate corrections for both the electronic losses and the losses caused by sample self-attenuation. Though correction for both kinds of loss is important, correction for the self-attenuation losses is more difficult. The emphasis of this report will be on the correction for self-attenuation in connection with appropriate physical standards to obtain accurate gamma-ray assays.

However, a few comments about the correction for electronic losses as it applies to high- and low-resolution detectors will be given here, and a short discussion will be given later on how this correction fits into the overall assay scheme. In general, the lower resolution detectors (certainly the various scintillators) are faster and the correction for electronic losses is so small that it can often be ignored or estimated with accuracy commensurate with the overall possibilities for accuracy in a given situation. For equal count rates, the high-resolution detectors will require a greater correction for electronic losses, but that correction can be made with high accuracy, thus making possible highly accurate assays.

\section{B. General Description of Assay Procedure}

If the raw data acquisition rate is multiplied by appropriate correction factors for both the rate-related electronic losses and for the sample self-attenuation, we may write

$$
\mathrm{TCR}=\mathrm{RR} * \mathrm{CF}(\mathrm{RL}) * \mathrm{CF}(\mathrm{AT})
$$

where

$$
\begin{aligned}
\mathrm{TCR}= & \text { total corrected rate, } \\
\mathrm{RR}= & \text { raw rate of data acquisition, } \\
\mathrm{CF}(\mathrm{RL})= & \text { correction factor for rate-related elec- } \\
& \text { tronic losses, and } \\
\mathrm{CF}(\mathrm{AT})= & \text { comection factor for self-attenuation in } \\
& \text { sample. }
\end{aligned}
$$

If the correction factors are properly defined and computed, TCR is the data acquisition rate that would have been observed if there were no electronic losses and if the sample were changed to a simpler shape (suck as a point or line) with the same gamma-ray emission but no self-attenuation. Thus computed, TCR is proportional to the mass of the isotope emitting the gamma ray of interest. We can then write

$$
\mathrm{TCR}=\mathrm{K} * \mathbf{M},
$$

where $M$ is the mass of the isotope being assayed and $K$ is a calibration constant. The calibration constant $K$ is determined by use of appropriate standard(s) and includes the effects of detector efficiency, subtended solid angles, and gamma-ray emission rates. The CF(AT) are determined so that the TCR for both unknown and standard are those that would have been observed if they had the same nonattenuating spatial configuration.

The above, in essence, constitutes this general approach to passive gamma-ray assay. Let us list the individual steps explicitly:

1. Measure the raw data acquisition rate.

2. Determine the correction for electronic losses.

3. Determine the correction for gamma-ray self-attenuation.

4. Compute the total corrected rate, which is proportional to the mass of the isotope being assayed.

5. Determine the constant of proportionality, the calibration constant, by use of appropriate physical standards, making sure that the TCR for both standards and unknowns represents the same nonattenuating geometrical shape in the same position with respect to the detector. 
It is clear that three quantities must be determined for both standards and unknowns: $R R, C F(R L)$, and CF(AT). The accuracy of an assay depends on the accuracy of each of the three factors that multiply together to give TCR. Both RR and $C F(R L)$ are relatively easy to determine accurately and will be discussed only briefly. The determination of CF(AT) is by far the most difficult and is the principal subject of this report.

\section{Brief Discussion of Data Acquisition Rate, Deadtime, and Pileup}

1. Determination of the Raw Data Acquisition Rate. Assuming that the experimental data are acquired by means of a high-resolution detector and a multichannel analyzer, we find the basic information is the areas $A$ of the full-energy spectral peaks of the gamma rays of interest and their statistical uncertainties $\sigma(A)$. The fullenergy peak area $A$ divided by the true time of acquisition TT yields the raw data acquisition rate $R R$.

Methods for extracting the full-energy peak areas from the spectra fall generally into two categories. The first category, applicable $\mathrm{m}$-inly to well-resolved peaks suffering no interference from nearby peaks, uses the sums of the channel counts in regions of interest (ROI) relating to the peaks. Usually, a single ROI brackets the peak, and one is assigned on each side of the peak to establish the correction that must be made to the peak ROI sum to compensate for the underlying Compton continuum. Such ROI schemes are quite simple, and it is straightforward to obtain the statistical uncertainty of the extracted areas. Though the scheme is simple in concept, careful attention must be given to the placing and the widths of the ROIs if accurate and consistent areas are to be obtained. References 2 and 3 give specific formulas that can be applied in using the ROI area scheme.

The second category of methods fits analytical functions to the full-energy peaks or groups of peaks. Such methods range from relatively simple ones that fit a single Gaussian function to a single well-resolved peak to very complex ones that fit a three- or four-component peak function to overlapping multiplets of several peaks. The complexity of the spectra expected in a given application will usually govern the choice of method to obtain the peak areas:

All methods for peak area determination work better on high-quality spectra, those without distortion caused by poor-quality detectors, poor-quality or improperly adjusted equipment, or excessive count rates. Knoll's book 4 is probably the most useful single-volume source of information on detectors, signal-processing electronics, and data analysis methods now available (1983), and it contains many references to more specialized works. Adams and Dams ${ }^{5}$ is also a good though older single-volume reference work on many aspects of gamma-ray spectroscopy. For information on the capabilities of the latest detectors and data acquisition equipment and for proper operation procedures, manufacturers' literature should be consulted.

High-quality spectra and appropriate peak area extraction methods consistently and carefully applied are important to accurate gamma-ray assays. Systematic errors of several per cent can easily creep in through sloppy data acquisition or careless peak area extraction methods. In general, greater errors can arise through using incorrect procedures or algorithms in the computation of the required correction factors from those peak areas.

2. The Correction for Deadtime and Pilewp. The corrections for data loss because of pileup and deadtime can be very significant. At high count rates in a highresolution detector, most of the potential information in a spectrum may be lost to those causes, requiring corrections as great as several hundred per cent. Thus, under some conditions the rate-related electronics losses may be as great as or greater than those caused by sample selfattenuation. The point here is that these electronics losses cannot be neglected if accurate, unbiased assays are desired. There are currently three types of methods for making the necessary corrections. All the methods assume that all spectral full-energy peaks suffer the same fraction of loss because of deadtime and pileup.

First, there are the purely electronic methods, most of them based on fast timing circuitry that detects piled-up events and extends the counting time sufficiently to correct for the combined effects of both pileup and deadtime. Such systems suffer limitations in the finite resolving time of germanium detectors (usually $\geq 0.5$ $\mu \mathrm{s})$ and in the first-order procedure normally used in extending the counting time. Generally, they are not adequate for use in assay systems where high accuracy is desired over a broad range of count rates. Recent work, particularly by Westphal, ${ }^{6}$ seems to remove the limitations and may offer the required accuracy at the price of a more complex data acquisition system. Additionally, if work must be done under conditions of both changing rates and spectral shape, methods such as Westphal's are the only ones with the required capabilitv.

A second commonly used procedure employs pulsers to inject into the spectrum a peak, which suffers nearly the same fraction of losses as do the gamma-ray peaks. Correction is made by comparing the number of pulses injected with the number appearing in the pulser peak. There are numerous implementations of the pulser method, some employing ordinary fixed-period pulsers, some using random pulsers, and some using pulsers in 
which the pulser rate is related to the input rate to the spectroscopy system. Good results can be obtained with all the implementations, but there are common difficulties relating to amplitude and/or rate stabilities of the pulsers and to the difficulty of inserting the pulses through the preamplifier without some degree of spectrum-distorting undershoot at the amplifier output. If a fixed-period pulser is used, as is most common, a correction must also be made for the fact that the pulser events, which neither pile up on themselves nor are lost because of their own deadtime, suffer somewhat smaller losses than the full-energy gamma-ray events.

The third method may be termed the reference source method, and at this time it seems most able to provide, with standard spectroscopy equipment, highly accurate corrections for the combined losses caused by deadtime and pileup. It is similar to the pulser method in that corrections are based on a reference peak in the spectrum, but the reference peak comes from a gamma-ray source fixed in position relative to the detector so that the detector sees a constant flux from the source. Stated differently, the reference gamma ray has a fixed fullenergy interaction rate (FEIR) in the detector. Based on the assumption that all the full-energy peaks in a spectrum suffer the same fraction of loss because of deadtime and pileup, the ratios of the areas of any other assay-related peaks to the reference peak area are independent of such losses. Assays can be based on those ratios without ever explicitly determining the actual fraction of loss. However, if the FEIR of the reference gamma ray is known (and it can be determined quite accurately by the pulser method) the actual magnitudes of the rates and corrections involved in an assay are easily computed.

These procedures and devices are not the subject of this report. However, those doing high-resolution gamma-ray assays must become as familiar and skillful with them as with the corrections for sample self-attenuation. A short conceptual definition of CF(RL) will be given, however, to show how it applies in Eq. (1). For any given sample-to-detector configuration, there will be a fixed rate of FEIRs in the detector for each gamma ray. Some fraction of the FEIRs will be included in the full-energy spectral peaks, most of the rest being lost to the combined effects of deadtime and pileup.* The correction factor for CF(RL) is determined so that the FEIRs are given by

$$
F E I R=R R * C F(R L) \text {. }
$$

\footnotetext{
*A small and variable fraction of full-energy events will fail to be recorded in the spectral full-energy peaks because of incomplete charge collection in the detector crystal. The magnitude of the problem can vary from negligible to significant depending on the quality of the detector.
}

Thus, Eq. (1) could be written also as

$$
T C R=F E I R * C F(A T) \text {. }
$$

Finally, remember that in high-quality spectra, assuming a constant spectral shape, the fraction of loss from the combined effects of pileup and deadtime is very nearly the same for all the full-energy peaks in the spectrum, and that this fact is the basis for all of the useful correction procedures now employed.

\section{General Discussion of CF(AT)}

1. Necessary Assumptions for Determination of CF(AT). Of the quantities that enter into a gamma-ray assay, the correction for the loss of information because of sample self-attenuation is generally the most difficult to determine accurately. In fact, in many situations it is impossible to determine the correction with any degree of accuracy at all, and in others the obtainable correction will be of limited value. Because of this, a discussion of the conditions for which accurate assays are and are not possible is given here.

In determining $\mathrm{CF}(\mathrm{AT})$, the basic question is, what fraction of the gamma rays of interest that are emitied by a sample in directions such that they could reach the detector in the absence of absorption or scattering actually do reach the detector? If for a given energy the sample material can be characterized for sufficiently small volume elements by a single linear attenuation coefficient $\mu^{\ell}$, the fraction of gamma rays of that energy escaping unmodified from the sample can, in general, be computed and the desired correction thereby obtained. Knowing or determining the sample $\mu^{k}$ is the key to

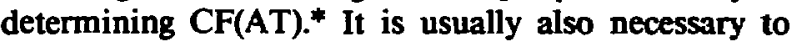
require that the individual particles of the gamma-rayemitting compound are so small that the self-attenuation within the individual particles is negligible (usually $<1 \%)$. This is particularly true when the sample is a mixture of a high-Z, high-density gamma-ray emitter (such as plutonium, uranium, and thorium) and a low$\mathrm{Z}$, low-density matrix (such as paper, rags, water, and acids). This requirement is necessary because though many fine-grained mixtures $(<100 \mu \mathrm{m})$ may have a well-defined and small $\mu^{2}$ for samples a few centimeters thick that correctly accounts for the attenuation of tlie gamma rays after they emerge from their parent particles, $\mu^{\ell}$ does not account for much of the attenuation within the parent particles.

*It will be assumed that the reader is familiar with the definitions of gamma-ray attenuation coefficients and with the basic laws governing their use in calculating gamma-ray attenuations in chemical compounds. References 4, 5, and 7 provió adequate background material for those needing it. 
Much study and experimental work have been devoted to defining conditions for which accurate assays are possible. The following two assumptions seem adequate to permit accurate gamma-ray assays when applied to the whole sample or a definable segment of it.

1. The mixture of gamma-ray-emitting material to be assayed and matrix material-everything but the assay material-is reasonably uniform and homogeneous in composition and of constant density.

2. The particles of gamma-ray-emitting compounds are small enough to make the self-attenuation within the individual particles negligible for the given assay situation and required accuracy.

These required assumptions will guarantee that the linear attenuation coefficient is single valued on a sufficiently microscopic scale that one can use it to accurately compute the necessary fraction of escaping gamma rays. They may seem quite restrictive and indeed do eliminate many heterogeneous, nonuniform samples from any hope of accurate gamma-ray assay. Howe ver, in other ways these assumptions are not restrictive. Most important, there are no restrictions at all on the chemical composition of the sample. All that is required is that the $\mu^{\ell}$ of the sample can be computed or measured. Because of this, unknown samples to be assayed need not have the same or even similar chemical compositions as the calibration standards. Note also that there are no basic assumptions about the size and shape of standards, though there are limitations. Standards and unknowns may also vary considerably in size and shape as will be discussed in detail below.

It is emphasized that the two assumptions given above concern only the material to be assayed. Even when the sample material itself meets those assumptions, an accurate assay may not be possible because of limitations and conditions on the density, size, and shape of the sample and on the material, thickness, and shape of the sample container. These conditions address such things as the necessity of the eswape of enough gamma rays from the sample and of having a sample shape simple enough to permit a reasonably straightforward computation of the CF(AT). More comment will be made on such conditions at appropriate places in this report.

The assumption of "reasonable uniformity" is admittedly vague and is difficult to define precisely. What constitutes reasonable uniformity will depend on the energy of the gamma ray being employed, the chemical composition of the sample, and the accuracy required. Some sample types will almost always adequately satisfy the assumbtions and some will almost never do so. In frequent cases what is known of the nature and composition of a class of material must be carefully considered and some computational work must be done to decide whether the material is reasonably uniforns for purposes of the desired assay.

It must be emphasized that it is the mass attenuation coefficients $\mu^{m}$ of the elements that impose the fundamental restrictions on the size, shape, composition, and density of samples that can be successfully assayed by gamma-ray methods. Those in charge of gamma-ray NDA measurements should be thoroughly familiar with the general trends, magnitudes, and dependencies of the mass attenuation coefficients as functions of atomic number and gamma-ray energy, and because of that importance, a few significant properties will be discussed here. The linear and mass coefficients are related by $\mu^{l}=\mu^{m} * \rho$, where $\rho$ is the actual spatial density of the elements in the sample. The mass coefficients are more fundamental than the linear ones because they are independent of the actual spatial density and state of the elements in physical compounds and mixtures.

Figure 1 shows mass attenuation coefficients vs energy for selected elements ranging from hydrogen ( $Z$ $=1)$ to plutonium $(Z=94)$. Qualitatively, the information in the graph defines nearly all the possibilities and limitations of passive gamma-ray NDA. Several important features should be noted. Between 1 and $3 \mathrm{MeV}$, the

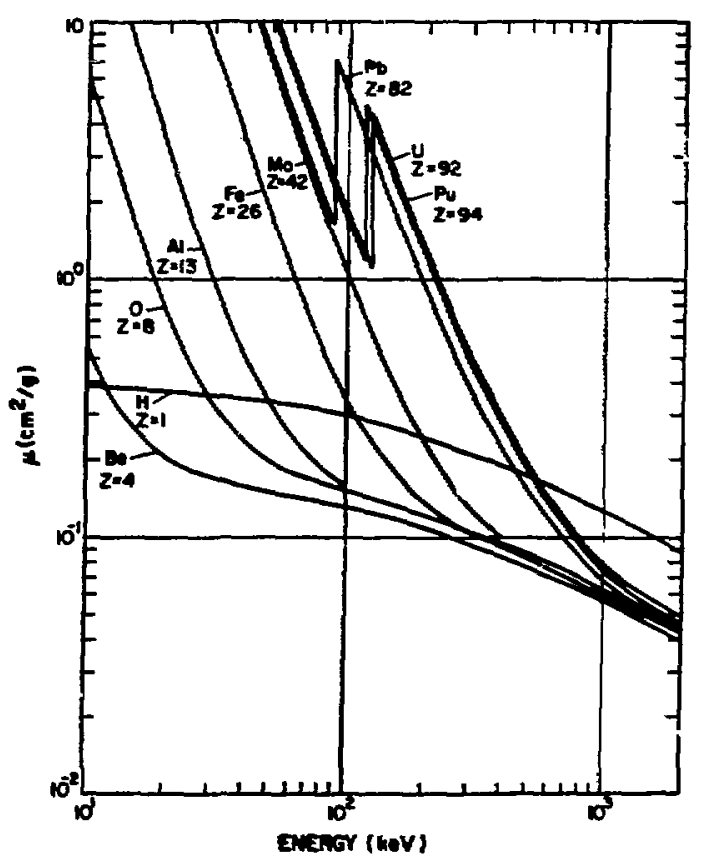

F5. 1. Toul mass attenuation coefficients (without wherent scattering contribution) vs enerty for nine elements rangins in atomic number $Z$ from $I$ to 94 . Source is Ref. 11. 
$\mu^{\mathrm{m}}$ for all elements are equal within about $\pm 20 \%$, and the average value is $\sim 0.05 \mathrm{~cm}^{2} / \mathrm{g}$. In this energy range, Compton scattering is the dominant attenuation process. If all the isotopes of interest had an adequately intense gamma ray in this range, gamma-ray assay would be much easier. Unfortunately, very few are so endowed. At low energies, the $\mu^{\mathrm{m}}$ of the high- $Z$ elements of interest become much larger than those of low- $Z$ materials, reaching values $\sim 20$ times higher near the $K$ absorption edges of uranium and plutonium. Both the high absolute values and the large ratios between highand low- $Z$ values make the quantitative assay of high- $Z$ materials by low-energy gamma rays difficult, and in frequent cases, even impossible. For example, a 2-mm thickness of uranium metal $\left(\sim 18.7 \mathrm{~g} / \mathrm{cm}^{3}\right)$ is essentially infinitely thick to the $185.7-\mathrm{keV}$ gamma ray, which is the principal emission of ${ }^{235} \mathrm{U}$. The transmission of that gamma ray through a $2-\mathrm{mm}$-thick layer of uranium is only $\sim 0.5 \%$. The quantity of ${ }^{235} \mathrm{U}$ in uranium metal objects thicker than $\sim 2 \mathrm{~mm}$ is therefore impossible to measure by detection of the $185.7-\mathrm{keV}$ gamma ray. Note, however, that $\mu^{\mathrm{m}}$ for uranium at $185.7 \mathrm{keV}$ is nearly six times larger than that of plutonium at $413.7 \mathrm{keV}$, the energy of the most useful gamma ray of ${ }^{239} \mathrm{Pu}$. This means that the assay of ${ }^{235} \mathrm{U}$ by its $185.7-\mathrm{keV}$ gamma ray is subject to considerably more stringent limitations on sample size, particle size, and uniformity than is the assay of ${ }^{239} \mathrm{Pu}$ by its $413.7-\mathrm{keV}$ gamma ray. Below $\sim 120 \mathrm{keV}$, gamma-ray assay may well be complicated by the fact that the sample $\mu^{\ell}$ can have discontinuities as a function of energy because of the absorption edges for the elements in the sample and also by the emission of $x$ rays by absorption at the edges. Below $\sim 80 \mathrm{keV}$, the $\mu^{\mathrm{m}}$ of most elements rise rapidly, making attenuation problems unmanageably severe for all but small samples of very small particle size. For assays of high-Z materials, useful applications of $<80-\mathrm{keV}$ gamma rays are limited to small samples of solution for accurate assays or to rough screening of larger packages of low- $Z$ materials for small quantities of high-Z gamma emitters.

Figure 2 is given as an aid in estimating self-attenuation for individual particles.* It gives the fraction of gamma rays escaping unscattered and unabsorbed from spherical sources as a function of the product $\mu^{\mathrm{m}} \mathrm{\rho D}$,

*The expression for the fraction of gamma rays escaping unscattered and unabsorbed from a sphere whose attenuation properties are characterized by $X=\mu^{\mathrm{m}} \rho \mathrm{D}$ is given by

$$
F=\frac{3}{2 X}\left[1-\frac{2}{X^{2}}+e^{-x}\left(\frac{2}{X}-\frac{2}{X^{2}}\right)\right]
$$

For proof of this expression, see: Ref. 8.

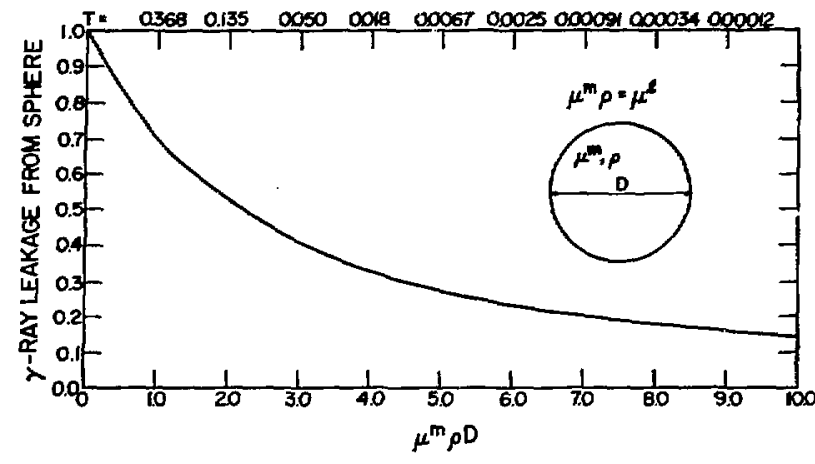

Fit. 2. Fractions of gamma rays escaping unscattered and unabsorbed from spherical gamma-ray-emitting particles as a function of $\mu^{\mathrm{m}} \mathrm{\rho D}$, where $\mu^{m}$ is the mass attenuation coefficient of the particles at the energy of interest, $\rho$ is the density of the particle, and $D$ is the diameter of the particle. Coherent (elastic) scattering has been neglected.

where $\mu^{m}$ is the mass attenuation coefficient for the source material, $\rho$ is the density of the material, and $D$ is the diameter of the sphere. As an example, for a 200- $\mu \mathrm{m}$ diam, $\rho=10$ particle of $\mathrm{UO}_{2}, \mu^{\mathrm{m}} \rho \mathrm{D} \simeq 0.28$, indicating that $\sim 10 \%$ of the $185.7-\mathrm{keV}$ gamma rays emitted are scattered with some energy loss or are completely absorbed within the particle.

Finally, having described some of the considerations that determine whether and to what degree a given sample material will meet the required assumptions for accurate gamma-ray assay, we should give some examples. First, of course, solutions by definition meet the criteria, assuming that there are no particulates or precipitates in the solution. Pure powders $\left(\mathrm{PuO}_{2}, \mathrm{UO}_{2}\right.$, $\mathrm{U}_{3} \mathrm{O}_{8}$, and so forth) almost always are suitable as are certain well-mixed scrap materials such as incinerator ash. High-temperature gas reactor (HTGR) coated fuel particles and HTGR-type rods come close to meeting the requirements, but assay results will be low by 5-10\% unless correction is made for the self-attenuation in the particle kernels. Small quantities of high-Z gamma emitters $(<10 \mathrm{~g})$ mixed with low-Z, low-density combustibles may meet the requirement if there are no agglomerations of the powder with significant self-attenuation. Large quantities of high- $Z$ powders (greater than about $100 \mathrm{~g}$ ) will almost surely create some significantly attenuating agglomerations when mixed with such combustibles. Among the worst cases are those with metal chips of high-Z, high-density metals or fuel pellets mixed with low-Z, low-density matrices; in these situations assays may well be low by factors of 2 or 3 or even more.

It must be emphasized that the degree to which samples of a given material satisfy the two assumptions is the most important factor in determining the potential accuracy of a gamma-ray assay of the material. 
Experience indicates, for example, that small samples of solution (up to a few tens of cubic centimeters) may be assayed with accuracies of a few tenths of a per cent. Samples of uniform, homogeneous powders of volumes up to a few liters have been assayed with accuracies approaching $1 \%$ in spite of significant density gradients. Larger containers of waste (for example, 30-gal. drums) rarely satisfy the assumptions well enough to allow errors of $<10 \%$, and as mentioned above, the error will be much worse for the extremely heterogeneous cases.

Having emphasized the errors caused by samples deviating from the required properties, we can fairly say that there are a few cases of samples that are heterogeneous, but in a regular known way, for which correct CF(AT) may be de termined. $^{9}$ HTGR fuel materials are an example. Such cases are rare, however, and the measured data must be supplemented by some prior knowledge of the sample composition.

Another important general fact about gamma-ray assays is that the results are almost always low when samples that do not satisfy the necessary assumptions are assayed in conjunction with calibration standards that do satisfy the assumptions. The reason for the low results is that the procedures that correctly determine the fraction of self-attenuation in acceptable samples underestimates that fraction in samples that fail to satisfy the required conditions. As noted, the error may be several hundred per cent in extreme cases. This fact causes one to be cautious about using gamma-ray methods to screen heterogeneous materials for possible criticality dangers.

2. Methods for Determining the Sample Linear Attenuation Coefficient. As before stated, if a sample satisfies the two assumptions and if its linear attenuation coefficient $\mu^{\ell}$ is known, we can compute both the fraction of unmodified gamma rays reaching the detector relative to those that could have reached it in the absence of self-attenuation and the desired correction factors. The basic problem, then, is to determine the sample $\mu^{2}$. Four principal methods have been employed, though one is really an evasion of the problem. Although the four procedures have been discussed together previously, ${ }^{10}$ it is well to do so again here.

a. Representative Standards. The oldest and still a frequently used (and abused) method is that of avoiding the issue by using representative standards. In this procedure a . $:$ of calibration standards is prepared as nearly identical as possible in size, shape, and composition to the unknowns with varying concentrations of the material to be assayed. The standards are counted in a fixed geometry to prepare a calibration curve, and the assay is accomplished by counting the unknowns in the same geometry and comparing the count directly with the calibration curve. This procedure will produce good results only if the unknowns and standards are sufficiently similar that the same concentration of assay material in each gives rise to the same sample $\mu^{\mathfrak{l}}$ and, therefore, to the same CF(AT). In other words, at the same concentration of assay material, the same fraction of gamma rays must escape from both sample and standard. The representative standard procedure usually also assumes that the pileup and deadtime losses are equal for equal concentrations of assay isotopes. This method is only applicable when the nature and composition of the assay samples are well known and essentially unvarying as is the case, for example, for some process solutions. The difficulty, if not futility, of trying to create representative standards for many categories of material is so obvious that it does not require discussion.

b. Computation from Knowledge of Composition. A second method exploits previous knowledge of the chemical composition, mass, and shape to compute the sample $\mu^{\mathfrak{l}}$ from which in turn the correction factor is computed. Sufficient prior knowledge to compute the sample $\mu^{\ell}$ does not necessarily mean that the assay result is known in advance. In many cases, the assay material is a small but unknown fraction of the total sample mass, and the sample $\mu^{\mathfrak{l}}$ is almost purely dependent on the matrix composition and mass, which is reasonably well known. The correction factor can therefore be computed, and especially when the highest accuracy is not necessary, this approach is useful. When only verification measurements are required on well-characterized materials, the approach is useful even when the assay material contributes significantly to the sample selfattenuation. Computation of the sample $\mu^{\ell}$ from knowledge of the chemical composition and densities is straightforward but must be done correctly. The necessary mass attenuation coefficients are reported along with discussion of the methods used to obsain the tabulated values and their uncertainties. ${ }^{11,12}$ Numerous texts discuss in detail the interaction of gamma rays with matter including the computation of linear attenuation coefficients from the known chemical composition and densities. Refercnces 4, 5 , and especially 7 are useful examples.

c. Gamma-Ray Intensity Ratios. Another method of determining $C F(A T)$ involves measuring the intensity ratio of gamma rays of two different energies from the same isotope in the sample and comparing it with the same ratio from a thin source of negligible self-attenuation containing the same isotope. This method is of limited use because, in general, the sample $\mu^{\mathbb{1}}$ is not uniquely related to the measured intensity ratios. Some prior knowledge of the nature of the sample is also 
required to obtain the actual correction factors. Furthermore, not all isotopes have a pair of gamma rays of the appropriate energies. Nevertheless, the method has proved useful in specific cases and has the potential for giving warning when the assumptions on uniformity and particle size are grossly violated.

d. Transmission Method. The fourth and most general method of obtaining the $C F(A T)$ involves the direct experimental measurement of the sample $\mu^{\ell}$ by measuring the transmission through the sample of a beam of gamma rays from an external source. From the fundamental law of gamma-ray attenuation, the transmission

$$
T=\exp \left(-\mu^{\ell} \mathbf{x}\right)
$$

where $\mathrm{x}$ is the thickness $c^{f}$.je sample. Solving for $\mu^{q}$, we obtain

$$
\mu^{\ell}=\frac{-\ln (T)}{x}
$$

As previously stated, this method requires no knowledge of the chemical composition or density of the sample, just the basic assumptions on uniformity and particle size, which were discussed above. As such, it is the most practical way to proceed in the ever-present "black box" cases. In fact, it is often the preferred method even when some knowledge of the sample composition is available, particularly when the best obtainable accuracy is desired. Let it be emphasized again that the experimentally measured sample $\mu^{\sharp}$ includes all the effects of chemical composition and density, which means that the calibration standards and the samples to be assayed need not have the same or even similar chemical compositions.

The transmission method also has the advantage of identifying those samples for which accurate quantitative assays are impossible because of excessive selfattenuation even though they satisfy the two basic assumptions. As the measured transmission becomes smaller, its precision deteriorates, and the precision of the sample $\mu^{\ell}$ computed from it also becomes worse, thus creating error in the computed value of $C F(A T)$. Depending somewhat on the sample, the energies involved, the particular equipment being used, and the allowable count times, the precision and accuracy of the measured transmission become unacceptably poor for values of transmission between 0.01 and 0.001 . Transmission values $\leq 0.001$ (perhaps even negative) almost always indicate an unassayable sample.

\section{FORMAL DEFINITION OF CORRECTION FOR SELF-ATTENUATION}

The definition of the correction factor for sample selfattenuation $\mathrm{CF}(\mathrm{AT})$ used in this report is implicit in the discussion of Sec. II, particularly in Eq. (4). It is appropriate, though, to give a generalized formal definition and make some remarks about a few particular forms that have proved useful.

\section{A. The General Definition}

Expressions for $\mathrm{CF}(\mathrm{AT})$ can be, and have been, formulated in a number of useful ways. The formulation adopted here has been prompted mainly by a preference for multiplicative correction factors and by a desire to obtain a corrected count rate that is directly proportional to the quantity of isotope being measured. It has proved convenient and useful for a number of years.

As indicated in Sec. II, it is useful to define CF(AT) with respect to a specified geometrical shape, which is often not that of the actual sample. The specified shape is usually simpler than the actual one, a point, for example. Let us then define $\mathrm{CF}(\mathrm{AT})$ by rearranging Eq. (4) to give, with only slight change in notation to make the concept more explicit,

$$
\mathrm{CF}(\mathrm{AT})=\frac{\text { FEIR }(\mu=0, \text { Spec Shape })}{\operatorname{FEIR}(\mu \neq 0, \text { Real Shape })},
$$

where

$\begin{aligned} \text { CF(AT) }= & \text { the correction for self-at- } \\ & \text { tenuation with respect to } \\ & \text { a particular specified } \\ & \text { shape, } \\ \text { FEIR }(\mu=0, \text { Spec Shape })= & \text { the FEIR that would have } \\ & \text { been measured if the } \\ & \text { sample were totally } \\ & \text { nonattenuating }(\mu=0) \\ & \text { and if it were changed to } \\ & \text { the specified shape, and } \\ \operatorname{FEIR}(\mu \neq 0, \text { Real Shape })= & \text { the actual measured } \\ & \text { FEIR from the sample. }\end{aligned}$

Now $C F(A T)$ is not computed in practice by dvidint $\operatorname{FEIR}(\mu=0$, Spec Shape) by the measured FEIR $(\mu \neq 0$, Real Shape). Indeed, FEIR( $\mu=0$, Spec Shape) is in reality the total corrected rate TCR of Eq. (4), which we seek to determine because it is directly proportional to the quantity of isotope being measured. 
The $C F(A T)$ is actually determined by computing the ratio of FEIR ( $\mu=0$, Spec Shape) to FEIR $(\mu \neq 0$, Real Shape) based on the known sample $\mu^{\ell}$, the sample geometrical configuration, and the position of the sample relative to the detector. Most often the expressions for the ratio are not integrable in terms of elementary functions, so numeric methods must be used. There are a few exceptions in which the expressions may be integrated and CF(AT) given in closed form, most of which will be discussed below.

Because CF(AT) is computed as the ratio of count rates, one does not generally need to know the detector efficiency for the computation.* Indeed, most often one can assume a point detector with equal efficiency for all angles of incidence, which considerably simplifies the computations. The point detector assumption is usually good when the distance between sample and detector is at least several times the maximum dimension of either detector or sample. If the sample-to-detector distance must be kept small for reasons of efficiency and the highest obtainable accuracy is required, the actual measured or calculated detector efficiency as a function of energy and position may be used. Cline describes a procedure for creating an efficiency function based on measurements of standard sources, which should be adequate for almost all requirements. ${ }^{13}$

\section{B. Useful Specified Shapes}

In 1983 , the most useful specified shapes were

1. the actual sample shape,

2. a point, and

3. a line.

If one has many samples of the same shape and size in a standard container and calibration standards in the same configuration, then the CF(AT) may be computed with respect to a nonattenuating sample of the same shape. However, remember that two nonattenuating sources of different shapes will not usually produce the same count rate per unit activity when centered at the same point with respect to the detector, assuming the samples have some well-defined center. This condition is obviously a consequence of the effects of the inverse square law, which must always be remembered, especially when standards and unknowns are of grossly

\footnotetext{
*This is not to say that the detector efficiency is not important. Indeed, the efficiency must be great enough to give adequate count rates for the particular assay situation and must be roughly known to estimate those rates. It is just that the detector efficiency does not usually enter explicitly into the deiermination of CF(AT).
}

different size and/or shape. Therefore, in all cases in which the whole sample is sufficiently uniform and homogeneous and of reasonable size, it is probably best to let the detector view the whole sample and to use the $\mathrm{CF}(\mathrm{AT})$ computed with respect to a nonattenuating point. As shown in the discussion of the general procedure in Sec. 1I, the TCR computed with the help of such $\mathrm{CF}(\mathrm{AT})$ are independent of the size and shape as well as of the chemical composition and thus allow the standards and the unknowns to be of different size, shape, and chemical composition. Remember, however, that for such assays to be accurate, the entire contents must be reasonably well represented by a single $\mu^{\ell}$.

Often samples have vertical gradients of density or vertical discontinuities of composition and density, the natural consequence of filling relatively narrow cylindrical or square containers of waste, scrap, or product from the top. As is to be expected, things tend to fall ints the containers in layers. In such cases, a single $\mu^{\ell}$ cannot adequately characterize the whole sample, but narrower layers or segments can be adequately characterized by a single $\mu^{\mathrm{R}}$ value. Assay accuracy can be improved by using a segmented scan in which the detector views the sample through a vertical collimator that defines relatively narrow horizontal segments, or layers, in which $\mu^{\ell}$ can be assumed constant. For such segmented scans, because of the effects of the collimator, it is best to compute the $\mathrm{CF}(\mathrm{AT})$ with respect to a nonattenuating line along the axis of the containers. In this way cylindrical samples may be accurately assayed with respect to standards of quite different diameters.

\section{DETAILED DISCUSSION OF CF(AT)}

\section{A. Important Parameters and General Trends}

In general, the correction factor for self-attenuation, CF(AT), is a function of many parameters. Those currently recognized as significant, listed roughly in decreasing order of importance, are

1. the $\mu^{\ell}$ of the sample material;

2. the volume and shape of the sample material;

3. the $\mu^{2}$ of the sample container;

4. the size and shape of the sample container, the shape of the container not necessarily being the same as that of the sample;

5. position and orientation of the sample relative to the detector; and

6. the size, shape, and efficiency of the detector.

The sample $\mu^{l}$ is listed first because the dependence of CF(AT) on it is usually strong and because for groups of 
samples of a standard size and shape, it is always the most variable parameter. This fairly lengthy list might suggest difficulty in accurately computing CF(AT) if all the parameters mentioned had to be known with high accuracy. Fortunately, however, in many situations the dependence of $C F(A T)$ on several of the parameters is mild, and very accurate values for them are not necessary. For example, when the sample-to-detector distance is at least several times the maximum dimension of the detector, the dependence of CF(AT) on the size, shape, and efficiency of the detector is often negligible. As another example, when the distance between a cylindrically shaped sample and the detector is at least several times the maximum dimension of either sample or detector, the CF(AT) is usually a strong function of the sample $\mu^{\ell}$, a mild function of sample dimensions and distance from the detector, and has negligible dependence on the detector size, shape, and efficiency.

The greatest simplifications in form and dependence oucur in what we shall call the far-field case, the one in which the maximum dimensions of both sample and detector are negligible conpared with the distance between them. In the far-field case, dependence on the inverse square law becomes negligible and all gamma rays reach the detector along essentially parallel paths. Thus, there is no dependence on detector size and shape, nor on small changes in the sample-to-detector distance, nor on saninple size except for the influence of size on the fraction of gamma rays escaping from the sample. Simple analytic expressions can be derived for several simple sample shapes. These forms are often useful for approximate work in situations not truly far field and for exhibiting general trends of CF(AT) for many assay situations. Indeed, the far-field situation is useful as a reference case against which to compare near-field cases.

In presenting $\mathrm{CF}(\mathrm{AT})$ values graphically to interpolate or to exhibit general trends, it is usually advantageous to plot $\mathrm{CF}(\mathrm{AT})$ vs the parameter of strongest dependence and to plot different curves for specific values of other parameters of significant but lesser importance. The parameter of strongest dependence is almost always the sample $\mu^{\ell}$, and if not of strongest dependence, then it is very often of greatest variability when considering a group of samples of fixed size and shape. The CF(AT) may always be plotted vs $\mu^{2}$; however, $\mu^{\ell}$ is often found by measuring the gamma-ray transmission $T$ through the sample and using the relationship $T=\exp \left(-\mu^{2} x\right)$, where $x$ is the sample dimension along which the transmission is measured. This expression suggests that CF(AT) could be plotted vs the product $\mu^{\ell} \mathrm{x}$ to avoid plotting separate curves for different values of $x$ or that the curves could be equivalently plotted vs $\ln (T)$, for $\ln (T)=-\mu^{l} x$. In fact, it is generally more convenient to plot $C F(A T)$ vs $\ln (T)$ because simpler analytic forms arise in the simple cases when those forms are expressed as functions of $T$. It is also useful to express analytic forms for $C F(A T)$ as functions of $T$ because of known limitations on the experimental determination of $T$.

As an illustration, consider the expression for CF(AT) for the far-field assay of a box-shaped sample viewed normal to a side. Neglecting any packaging, the expression (which shall be derived later) is

$$
C F(A T)=\frac{-\mu^{l} x}{\left[1-\exp \left(-\mu^{2} x\right)\right]},
$$

where $x$ is the the thickness of the sample along the normal to the detector. Using $T=\exp \left(-\mu^{2} x\right)$, we can write

$$
C F(A T)=\frac{-\ln (T)}{(1-T)}
$$

which is a very simple expression, indeed the simplest of any $c f$ the exact analytic expressions known. For $T \ll$ 1 , we see that $C F(A T) \simeq-\ln (T)$, so a plot of $C F(A T)$ vs $\ln (T)$ is nearly linear. Figure 3 gives a plot of CF(AT) = $-\ln (T) /(1-T)$. It also gives $C F(A T)$ vs $\ln (T)$ for cylindrically and spherically shaped samples where $T$ is measured across the diameter of the samples. All the

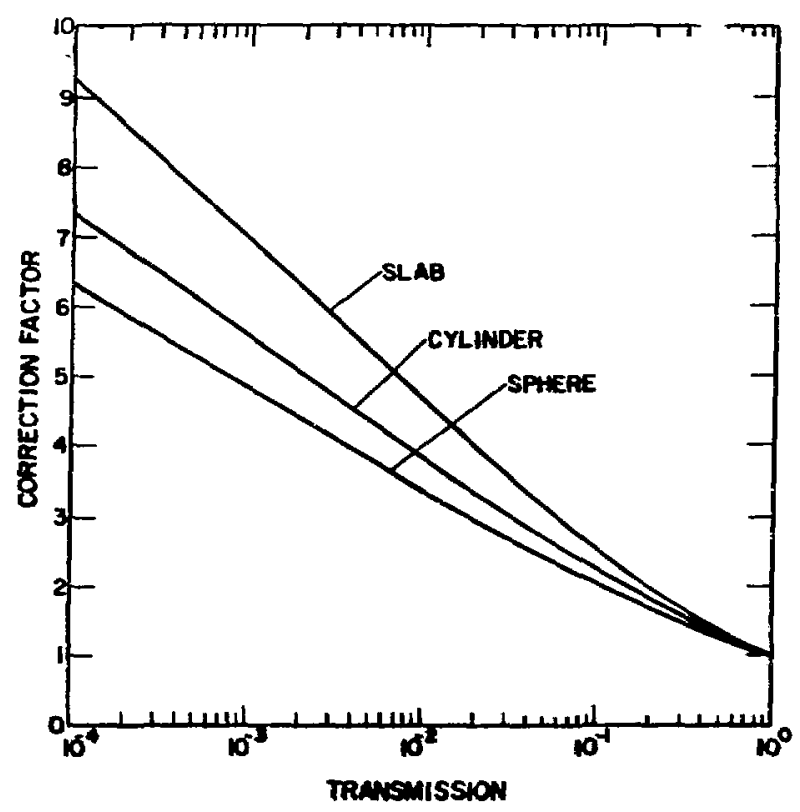

F2. 3. Far-field correction fectors for sibb, cylindical, and spherical samples as a function of transmiction. The tranemiscion is meaned normal to the froe of the stab ample and alons dianeter of the cyliadrical and spherical samples. 
cases are seen to be of the form $C F(A T) \simeq-k \ln (T)$ for $T \ll 1$. This approximate $\ln (T)$ dependence is rather general for most assay geometries and is very useful to keep in mind.

\section{B. Analytic Far-Field Forms for CF(AT)}

In general, when writing integral expressions for $\mathrm{CF}(\mathrm{AT})$ in the near field, we cannot integrate the expressions in terms of elementary functions to yield analytic expressions. In the far field, expressions have
The parameters $\rho, I$, and $\mu^{l}$ are constant, whereas $\varepsilon$ and 1 are functions of position. The integrations are over the total volume $V$. It is the exponential term in the denominator that, for most sample shapes and geometrical configurations, gives rise to expressions that cannot be integrated in terms of elementary functions.

Now let us write the specific expression for the configuration shown in Fig. 4, a parallelepiped of dimensions $X, 2 Y$, and $2 Z$ centered on the coordinate axes as indicated with a detector viewing the sample along the $x$-axis at a distance $D$ from the sample. The expression is

$$
C F(A T)=\frac{\int_{-Z}^{Z} \int_{-Y}^{Y} \int_{0}^{X} \rho I \varepsilon(x, y, z) d x d y d z}{\int_{-Z}^{Z} \int_{-Y}^{Y} \int_{0}^{X} \rho I \varepsilon(x, y, z) e^{-\mu_{r}(x, y, z)} d x d y d z},
$$

been derived for three simple sample geometries: box shaped (rectangular parallelepipeds), cylindrical, and spherical. These expressions will be given and discussed; the derivation will be given for the box-shaped samples. As a means of comparing the three cases, Fig. 3 gives the far-field $\mathrm{CF}(\mathrm{AT})$ for all three sample shapes as a function of $\ln (T)$, and Table I gives numeric values for the three cases over a wide range of transmission.

1. Box-Shaped Samples. This is the simplest case and the only one for which a simple derivation exists. Because the derivation is both simple and instructive, it will be given here. From Eq. (7), we can write the general expression for $\mathrm{CF}(\mathrm{AT})$ with respect to a nonattenuating sample (specified shape same as real shape) in integral form as

$$
C F(A T)=\frac{\int_{v} \rho I \varepsilon d V}{\int_{v} \rho I \varepsilon \exp \left(\mu^{2} r\right) d V}
$$

where

$$
\begin{aligned}
& \rho=\text { spatial density of the isotope being assayed } \\
& \left(\mathrm{g} / \mathrm{cm}^{3}\right),
\end{aligned}
$$

where the explicit dependence of $\varepsilon$ and $r$ on $x, y$, and $z$ is indicated and where $d x d y d z=d V$. Now $I$ is a constant for a given isctope, and by virtue of the fundamental assumptions on uniformity, we can assume $\rho$ and $\mu^{\ell}$ are also constant. In addition, the assumption of far-field conditions is equivalent to assuming $\varepsilon(x, y, z)$ is also a constant. Now note that for the configuration given in Fig. $4, r=(x-x) / \cos \theta$, where $x$ is the $x$-coordinate of the volume element and

Table I. Far-Field Correction Factors For Slab, Cylinder, and Sphere as Functions of Transmission

\begin{tabular}{cccc}
\hline Transmission & Slab" & Cylinder & Spluere \\
\hline 1.0000 & 1.000 & 1.000 & 1.000 \\
0.8000 & 1.116 & 1.097 & 1.006 \\
0.6000 & 1.277 & 1.231 & 1.202 \\
0.4000 & 1.527 & 1.434 & 1.376 \\
0.2000 & 2.012 & 1.816 & 1.701 \\
0.1000 & 2.558 & 2.238 & 2.054 \\
0.0500 & 3.153 & 2.692 & 2.431 \\
0.0200 & 3.992 & 3.326 & 2.956 \\
0.0100 & $\mathbf{4 . 6 5 2}$ & 3.826 & 3.370 \\
0.0010 & 6.915 & 5.552 & 4.005 \\
0.0001 & 9.211 & 7.325 & 6.283 \\
\hline
\end{tabular}

Tranedecion newenl to ewrice.

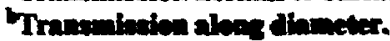




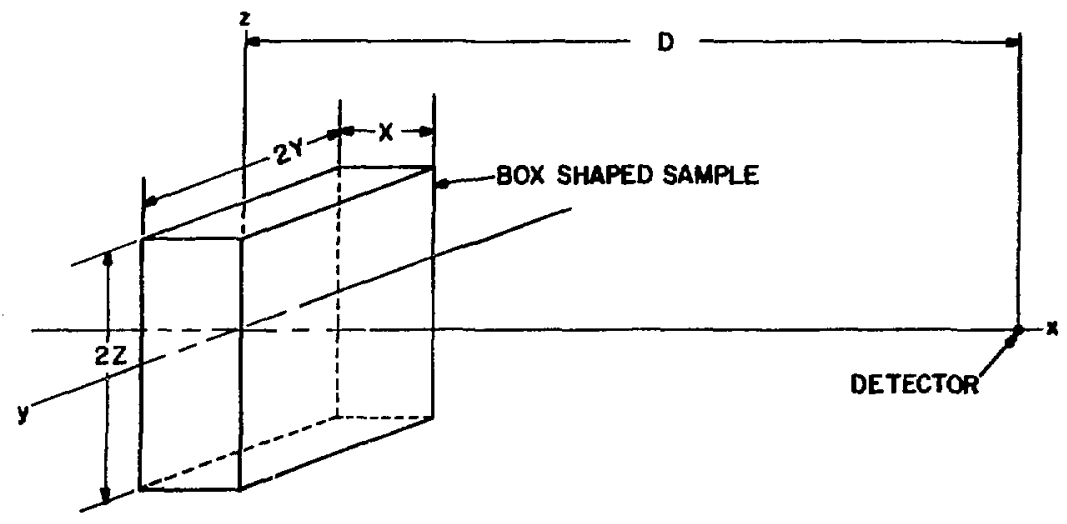

Fis. 4. Counting geometry for a slab-shaped sample with coordinates and dimensions for use in deriving the far-field correction factor.

$$
\cos \theta=\frac{(D-x)}{\sqrt{(\Sigma-x)^{2}+y^{2}+z^{2}}},
$$

This evaluates to

$$
\mathrm{CF}(\mathrm{AT})=\frac{\mu^{\ell} \mathrm{X}}{1-\mathrm{e}^{-\mu^{2} \mathrm{X}}},
$$

where $y$ and $z$ are the other coordinates of the volume element. Again, because of the far-field assumption, we have $D \gg X, Y$, and $Z$, and therefore $\cos \theta \simeq 1.0$ and : $\simeq(X-X)$. With these simplifications arising from the far-field geometry, the expression reduces to which is identical to Eq. (8) and easily transforms to Eq. (9). As noted above, this is a simple expression with the approximate $\ln (T)$ dependence for $T<1$. It is also useful in many practical situations that are not real far-

$$
C F(A T)=\frac{\rho I \varepsilon \int_{-Z}^{Z} \int_{-Y}^{Y} d y d z \int_{0}^{X} d x}{\rho I \varepsilon \int_{-Z}^{Z} \int_{-Y}^{Y} d y d z \int_{0}^{X} e^{-\mu^{l}(X-x)} d x},
$$

After the obvious cancellations, we have

$$
C F(A T)=\frac{\int_{0}^{x} d x}{\int_{0}^{x} e^{-\mu^{2}(x-x)} d x}
$$

field cases but for which this expression will provide values that are accurate within a few per cent.

2. Cylindrically Shaped Samples. For a cylindrically shaped sample viewed by the detector along a diameter in the far field, it has been shown that ${ }^{14}$ 


$$
C F(A T)=\frac{1}{2} \frac{\mu^{\ell} D}{\left[I_{1}\left(\mu^{\ell} D\right)-L_{1}\left(\mu^{\ell} D\right)\right]}
$$

where

$L_{1}=$ the modified Struve function of order 1 , $I_{1}=$ the modified Bessel function of order 1,

$\mathrm{D}=$ the sample diaineter, and where as usual,

$\mu^{\ell}=$ the sample linear attenuation coefficient.*

Again, the expression is very compact, but it is inconvenient to use because of the necessity for finding or computing values for the Struve and Bessel functions.

Inasmuch as ihe transmission across a diameter of a cylinder is given by $T=\exp \left(-\mu^{\ell} D\right)$, we can write the expression for $\mathrm{CF}(\mathrm{AT})$ as

$$
C F(A T)=\frac{1}{2}\left[\frac{-\ln (T)}{I_{1}[-\ln (T)]-L_{l}[-\ln (T)]}\right] .
$$

Equation (15) was used to generate the curve for a cylinder in Fig. 3. Note that the CF(AT) for a cylinder are a little less than those for a slab or box-shaped sample, a situation we can undersiand intuitively by comparing a square sample with a cylindrical one of th: same $\mu^{\ell}$ and whose diameter is equal to the side of the square. In the cylindrical sample, fewer gamma rays must penetrate the maximum thickness of material in escaping from the sample; hence, the fraction escaping is greater and the $\mathrm{CF}(\mathrm{AT})$ is smaller.

*As given in Ref. is (p. 375), the series expansion for the modified Bessel function of order $v$ and argument $z$ is given by

$$
I_{v}(Z)=\frac{Z}{2} \sum_{K=0}^{\infty} \frac{\left(Z^{2} / 4\right)^{K}}{K ! \Gamma(v+K+1)} .
$$

Also in Ref. 15 (p. 498), the series expansion for the modified Struve function of order $v$ and argument $Z$ is given as

$$
L_{v}(Z)=\left(\frac{Z}{2}\right)^{v+1} \sum_{K=0}^{\infty} \frac{(Z / 2)^{2 K}}{\Gamma(K+3 / 2) \Gamma(K+v+3 / 2)} .
$$

Values for the difference $\left[I_{1}(X)-L_{1}(X)\right]$ are also tabulated in Ref. 15 (p. 501) for arguments covering transmissions as low as 0.005 .
3. Spherically Shaped Samples. For a spherical : ple in the far field, the correction factor can be shor be $^{8}$

$$
\begin{aligned}
C F(A T)= & \frac{3 / 2}{\mu^{\ell} D}\left\{1-\frac{2}{\left(\mu^{\ell} D\right)^{2}}\right. \\
& \left.\left.+e^{-\mu^{\ell} D}\left[\frac{2}{\mu^{l} D}+\frac{2}{\left(\mu^{l} D\right)^{2}}\right]\right\}\right)^{-1},
\end{aligned}
$$

where $\mathrm{D}$ is the diameter of the sphere and as usual the sample linear attenuation coefficient. Obviou using $\ln (T)=-\mu^{\ell} \mathrm{D}$ we can write the expression as

$$
\begin{aligned}
\mathrm{CF}(\mathrm{AT})= & \left(\frac { 3 / 2 } { [ - \operatorname { l n } ( \mathrm { T } ) ] } \left\{1-\frac{2}{[\ln (\mathrm{T})]^{2}}\right.\right. \\
& \left.\left.+\mathrm{T}\left[\frac{2}{[-\ln (\mathrm{T})]}+\frac{2}{[\ln (\mathrm{T})]^{2}}\right]\right\}\right)^{-1}
\end{aligned}
$$

This expression is also plotted against In (T) in Fig The CF(AT) for a sphere in the far-field case are clea smaller than those for either the parallelepiped cylinder. Again, the trend is intuitively obvious comparing a sphere of diameter D with a cylinder diameter $D$ and a cube of side $D$. On the average, 1 gamma rays travel shorter distances to escape from 1 sphere than from either the cylinder or the cube. Th the fraction escaping is greater and the CF(AT) : smaller. Spherical samples are rarely mer in practice, $t$ the reciprocal of the expression for CF(AT) gives 1 fraction of gamma rays escaping from spherical partic and is useful in deciding whether a given particula containing sample adequately meets the required sumption on particle size. Written in terms of the $m$ a 
attenuation coefficier. $\mu^{m}$ and the density $\rho$ of the particle material, the fraction of gamma rays escaping is given by the sample diameter, a two-dimensional model in which a point detector views a circular sample may suffice. Clearly, if complicated geometries must be considered,

$$
F=\frac{3 / 2}{\mu^{m} \rho D}\left\{1-\frac{2}{\left(\mu^{m} \rho D\right)^{2}}+e^{-\mu^{m} \rho D}\left[\frac{2}{\mu^{m} \rho D}+\frac{2}{\left(\mu^{m} \rho D\right)^{2}}\right]\right\}
$$

Figure 2 was generated from this expression. As pointed out there, a $200-\mu \mathrm{m}$-diam, $\rho=10$ spherical particle of $\mathrm{UO}_{2}$ absorbs $\sim 10 \%$ of the $186-\mathrm{keV}$ gamma rays emitted within it. The reason that the assumption on particle size is necessary is that a $\mu^{\ell}$, which accurately describes the attenuation of a beam of gamma rays passing through a heterogeneous layer of material (both beam width and layer thickness much greater than the maximum particle size), says little about any absorption occurring within the individual emitting particles when nigh density, high- $Z$ emitters are contained in a lowdensity, low- $Z$ matrix. Thus, for example, in a sampie containing a dilute mixture of $200-\mu \mathrm{m}-\mathrm{diam} \mathrm{UO}_{2}$ particles in a graphite matrix, a transmission measurement will determine a $\mu^{\ell}$, which describes mostly what happens after the gamma rays leave the particles but corrects for little of the $\sim 10 \%$ loss within the emitting particles themselves. An $\sim 10 \%$ assay error is likely if the samples are being assayed with respect to standards that meet the assumption on particle size.

\section{Numeric Computation in the Near Field}

1. General Discussion. As mentioned above, for most if not all near-field situations in which inverse square dependence must be put into the integral expressions explicitly, the resulting expressions cannot be (or at least have not been) integrated in terms of elementary functions. As a result, numeric methods must be used, which almost automatically implies the use of computers. However, even with the power and availability of modern computers, it is good to minimize the effort required for the computation, which means that the simplest adequate model should be used to describe the assay situation. As before mentioned, it is often possible to simplify the computations by assuming a point or line detector with efficiency independent of angle of incidence. Other simplifications are also sometimes possible. For example, for assay of a cylindrical sample whose height is equal to or less than its diameter, and the sample-to-detector distance is at least several times very powerful Monte Carlo-type photon transport codes requiring the use of large computers can be used. However, more ofien the situation encountered in the NDA of simply shaped samples can be handled with simplified models and straightforward one-, two-, or three-dimensional numeric integration methods using relatively simple codes and quite small computers. Remember always that the accuracy of gamma-ray NDA is often determined more by the nonuniformity and inhomogeneity of the samples than by the lack of detailed accuracy in the CF(AT) computations. In such cases, perfectly adequate correction factors can often be obtained by a simple approximate model.

Clearly, if it takes minutes or even hours to compute a single value of $\mathrm{CF}(\mathrm{AT})$, then it will be advantageous to compute $\mathrm{CF}(\mathrm{AT})$ for a given container and assay geometry for a number of values of $T$ (or equivalently $\left.\mu^{\ell}\right)$ and to interpolate between them. That interpolation could clearly be done in several ways, some of which will be mentioned below.

For much work, simple approximate analytic forms exist that give adequately accurate values for $\mathrm{CF}(\mathrm{AT})$ over reasonable ranges of transmission (or $\mu^{k}$ ). A few such forms will be pointed out below. The adequacy of a particular approximate expression can be determined by comparison with more accurate numeric computations based on a suitable model. In connection with the discussion of the approximate forms, it will be pointed out and discussed in some detail that it usually does not matter if CF(AT) is in error by a constant multiplicative factor over the range of transmission concerned.

An unfortuniate aspect of computing $C F(A T)$ by numeric methods is the difficulty of computing the precision of $\mathrm{CF}(\mathrm{AT})$ as a function of the precision of the transmission value. The approximate analytic forms often provide the capability to derive sufficiently correct analytic expressions for the precision of CF(AT). This, too, will be discussed below.

2. A Useful One-Dimensional Model. A common assay geometry is that in which a germanium detector views a bottle of solution from beneath. Both detector 
and sample are generally well approximated by right circular cylinders. Assume that the axes of symmetry of the bottle and the detector coincide and that the detector radius is $r_{\mathrm{d}}$, the sample radius is $r_{\mathrm{s}}$, the sample depth is $\mathrm{D}$, and the distance from sample to detector is $\mathrm{d}$ (Fig. 5). Now if $d$ is a few times greater than both $r_{d}$ and $r_{z}$, no gamma ray from the sample will impinge on the detector at angles greater than $\sim 10^{\circ}$ from the common axis. Inasmuch as $\cos \theta \geq 0.95$ for angles $<19^{\circ}$, it is clear that no gamma ray from anywhere in the sample will travel more than a few per cent greater distance on its way to the detector than those that travel parallel to the common centerline. With this in mind, it is apparent that the essence of the assay situation can be described by a one-dimensional model consisting of a point detector and a line sample of "depth" $D$ and linear attenuation coefficient $\mu^{\ell}$ separated from the detector by a distance $d$ as indicated in Fig. 6. This model contains in a nearly correct way the inverse square law effects and the gamma-ray attenuation effects, which are the main influences on the CF(AT).

Having adopted the model, we give the CF(AT) with respect to the nonattenuating sample according to the definition of Eq. (7) by

$$
C F(A T)=\frac{\int_{0}^{D} \frac{d x}{(d+x)^{2}}}{\int_{0}^{D} \frac{e^{-\mu^{\ell} x} d x}{(d+x)^{2}}}
$$

where all constants pertaining to the detector efficiency and gamma-ray emission rates have canceled. The numerator immediately integrates to $D /[d(d+D)]$, but as simple as the denominator appears to be, it cannot be integrated in terms of elementary functions. However, it can be written as a sum in a simple way. The expression for $C F(A T)$ then becomes

$$
C F(A T)=\left[\frac{D}{d(d+D)}\right] / \sum_{I=1}^{N} \frac{e^{-\mu^{2}(I-0.5) \Delta x}}{[d+(I-0.5) \Delta x]^{2}},
$$

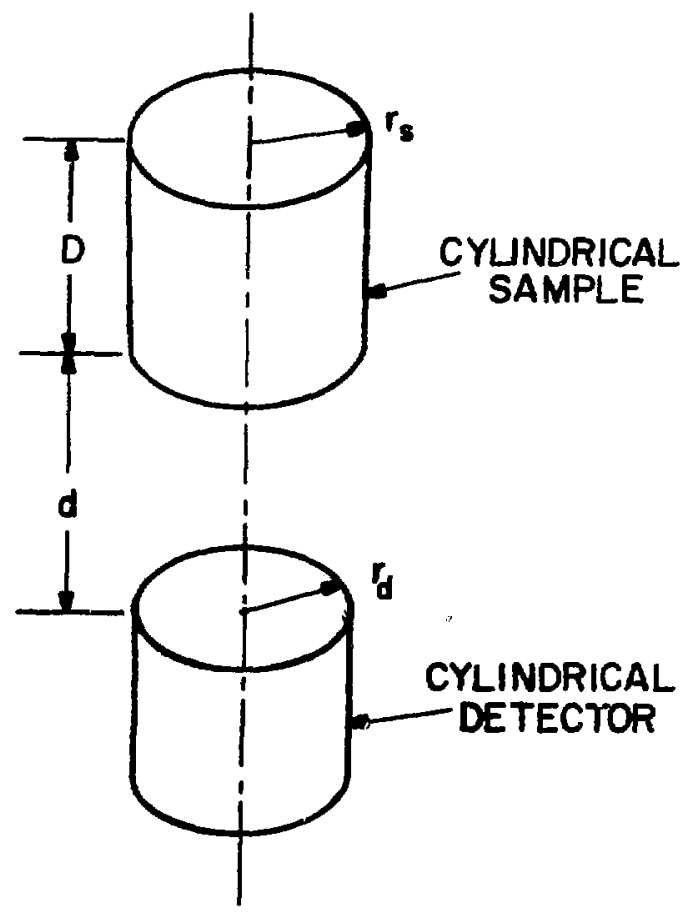

Ft. 5. Commonly used vertical astay seometry for which a one-dimensional model is approprinte for computins CF(AT).

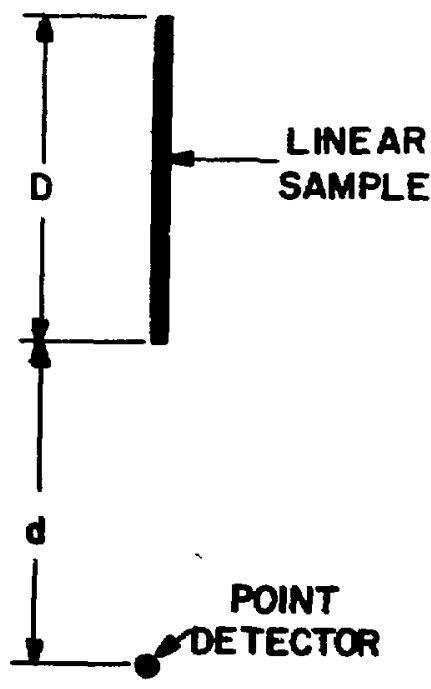

F2. 6. One-dimensional model for computing CF(AT). The detector is essumed to be a point, and the sample is ssaumed to be a line of kength $D$ with linear atienuntion coeficient $\mu^{2}$, which is separated from the point detector by distance $d$. 
where $\Delta x=D / N$ and $N$ is the number of intervals into which the sample depth is divided for the numeric integration. Generally, taking $N \simeq 100$ will give the result of the integral to $<0.1 \%$, which takes negligible execution time on almost any computer-based system. The numeric integration could, of course, be done with better accuracy by Simpson's rule or other more elegant methods in fewer steps. Equation (21) has been left in the form given to make as clear as possible the functional dependence of $C F(A T)$ on the parameters $d, D$, and $\mu^{l}$ and the equivalence of the integral and the sum. Of the two geometrical parameters $d$ and $D$, the latter is well defined as the depth of the sample. The parameter d, however, is less well defined, nominally the distance from the detector to the sample. However, in real detectors of finite volume, $d$ is not well defined because the gamma rays interact throughout the volume of the detector and because even the average interaction depth is a function of energy, a consequence of the variation of $\mu^{m}$ for germanium with energy. One might then ask how d is chosen to get the correct answers to a given situation. Experience has shown that if the nominal value of $d$ is at least a few times $D$, then with the help of a set of standards covering a wide range of $\mu^{\ell}, d$ can be adjusted to give values of $C F(A T)$ such that the corrected rate per unit activity is nearly constant over a wide range of $\mu^{\ell}$ (or alternatively, of course, a wide range of the transmission $T$ of the sample). By so adjusting $d$, compensa- tion is made not only for the imprecisely known sampleto-detector distance but also for the deviation of the one-dimensional model from the actual three-dimensional assay geometry.

Figure 7 shows results of a measurement exercise using the procedure just described to determine the appropriate $\mathrm{CF}(\mathrm{AT})$. The samples were 25 -m\& solutions of depleted uranium nitrate in flat-bottomed bottles of $10-\mathrm{cm}^{2}$ area, thus making the samples right circular cylinders of solution $3.57 \mathrm{~cm}$ in diameter and $2.5 \mathrm{~cm}$ deep. The uranium concentration varied from 5 to 500 $g / \ell$, and all the samples were spiked with an equal amount of ${ }^{75} \mathrm{Se}$. The detector crystal was $\sim 4.0 \mathrm{~cm}$ in diameter and $\sim 4.0 \mathrm{~cm}$ long. The corrections for electronic losses, $\mathrm{CF}(\mathrm{RL})$, varied by only $\sim 10 \%$, whereas the corrections for gamma-ray attenuation, CF(AT), varied by $\sim 275 \%$ for the results on the $136.0-\mathrm{keV}$ gamma ray of ${ }^{75} \mathrm{Se}$, which are presented in Fig. 7 . The lower part of Fig. 7 shows the magnitudes of CF(RL) and $C F(A T)$ as functions of uranium concentration; the sample transmission $T$ is also indicated for the nine samples used. Remember that because of the $2.5-\mathrm{cm}$ sample depth, for any sample $\mu^{\ell}=[-\ln (T) / 2.5] \mathrm{cm}^{-1}$.

Because each sample had identical amounts of ${ }^{75} \mathrm{Se}$, the corrected $136.0-\mathrm{keV}$ rate should have been equal for all samples. The upper part of the figure gives the fractional deviation of the corrected rates from the average of all and indicates the typical precision of the
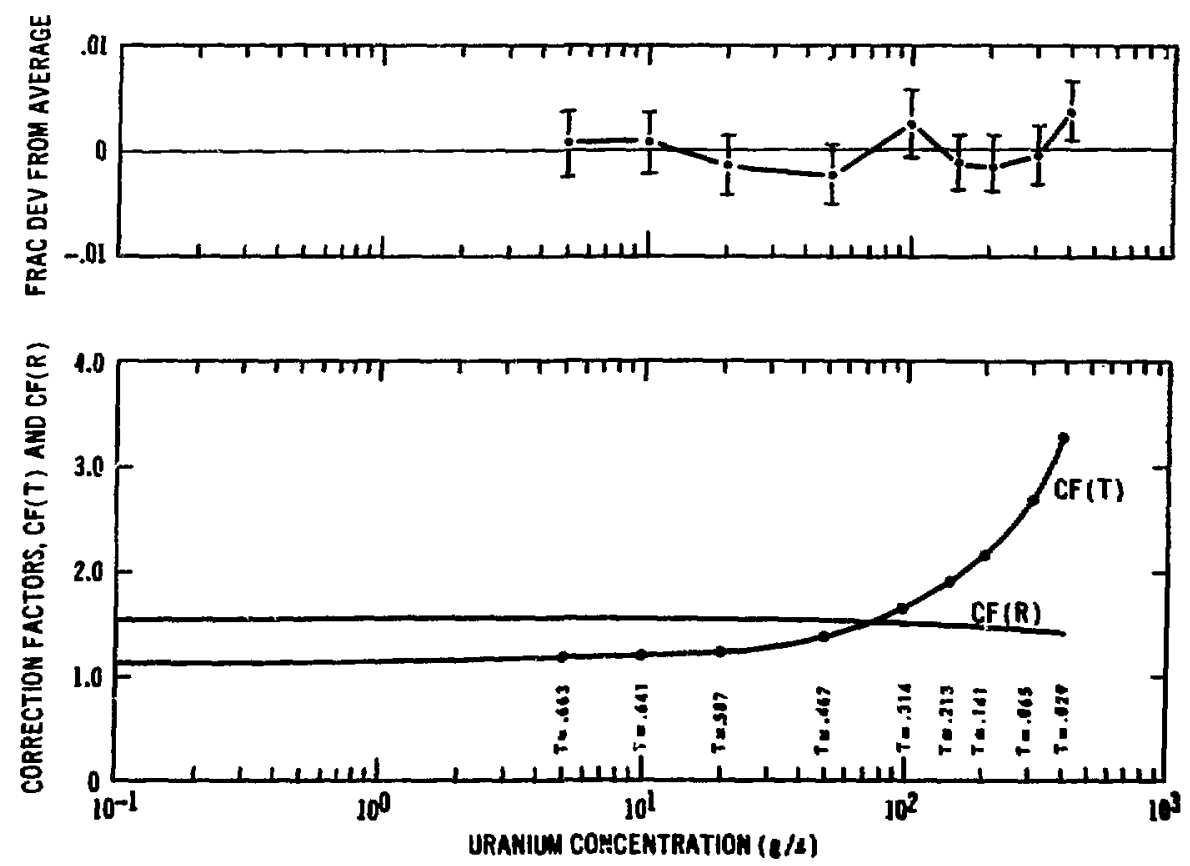

Fl. 7. Results of a measurement exercise desi med to test the usefulness of a one-dimensional model for computins $\mathrm{CF}(\mathrm{AT}) . \mathrm{CF}(\mathrm{AT})$ is indicated as a function of transmission $\mathrm{T}$ simply by CF(T). 
measurements. As can be seen, all the corrected rates are within about $\pm 0.5 \%$ of the average even though the CF(AT) changes by $\sim 275 \%$, whereas the $C F(R L)$ remains constant to within $\sim 10 \%$. This is a good example of how a very much simplified model can give good results for $\mathrm{CF}(\mathrm{AT})$ for quite complex assay geometries, particularly if there is one adjustable parameter whose value can be optimized with the help of suitable physical standards. In this case, the actual distance of the sample bottom to the average interaction depth in the detector was $\sim 8 \mathrm{~cm}$, and the adjusted value was $9.0 \mathrm{~cm}$. Qualitatively, the pure one-dimensional model will give values of $C F(A T)$ that are a little low compared with the correct three-dimensional model with the same $d$ and D. This is because in the actual assay geometry the gamma rays on the average will pass through slightly greater thicknesses of solution than in the one-dimensional model and thus require slightly greater corrections. Increasing $d$ increases $C F(A T)$ overall and also increases the CF(AT) more for lower values of $T$ relative to those of higher $T$ values as required. Hence, the value of $d$ used in computations will usually be a little higher than the physical value, the difference depending on the particular assay geometry.

If a set of solution samples has fixed diameter and position relative to the detector but has variable but determinable depths, one would prefer to compute the $\mathrm{CF}(\mathrm{AT})$ with respect to a nonattenuating point so that the corrected rates from all the samples can be directly compared. If the nonattenuating point is placed at the bottom of the idealized sample, a distance $d$ from the point detector, the expression for the CF(AT) becomes

$$
C F(A T)=\frac{\int_{0}^{D} \frac{d x}{d^{2}}}{\int_{0}^{D} \frac{e^{-\mu^{2} x} d x}{(d+x)^{2}}}
$$

The ratio between the $\mathrm{CF}(\mathrm{AT})$ with respect to the nonattenuating point and the $C F(A T)$ with respect to the nonattenuating sample is $(1+D / d)$, independent of $\mu^{\ell}$, as can be seen by taking the ratio of Eq. (22) to Eq. (20). For a set of samples of the same shape and counting geometry, it obviously does not matter which form for CF(AT) is used; but if sample depths vary, the CF(AT) with respect to the nonattenuating point must be used because the observed count rate from two nonattenuating linear samples of the same activity but different lengths would be different. These remarks are intended to emphasize the general rule that all CF(AT), for both standards and unknowns, should be computed with respect to the same nonattenuating shape so that the corrected rates might be directly comparable.
3. A Useful Two-Dimensional Model. Another common assay geometry is that in which a detector views a cylindrical sample from the side along a ray normal to the sample axis, usually centered along the sample depth (Fig. 8). Now if the sample depth is, for example, less than or equal to the sample diameter and if the distance from the detector to the sample center is at least several times the sample diameter, then a simple two-dimensional model can often be used to compute adequately correct values of CF(AT). The model consists of a point detector at a distance $D$ from the center of a cylindrical sample of radius $R$ and zero height. The use of a point detector implies that the full-energy interaction efficiency for the detector is essentially constant for gamma rays originating at any point within the sample volume. This assumption is almost true if $D$ is at least several times both the sample diameter and the detector diameter. Figure 9 shows the geometry of the two-dimensional model and indicates the distances that must be determined and the variables in terms of which they are expressed. The parameters $D$ and $R$ define the geometry. The distances that must be determined in terms of $D$ and $R$ and the coordinates of the area elements $r$ and $\theta$ are

- $L$, the distance from the area element to the point detector, and

- $t$, the distance from the volume element to the cylinder edge along $L$.

From the law of cosines, $L$ is found directly as

$$
L=\sqrt{D^{2}+r^{2}-2 D r \cos \theta}
$$

The expression for t is much more complex and could be written in various ways; one way follows. If the angle $\gamma$ is known, then from the law of cosines,

$$
t=\sqrt{R^{2}+r^{2}-2 r R \cos \gamma}
$$

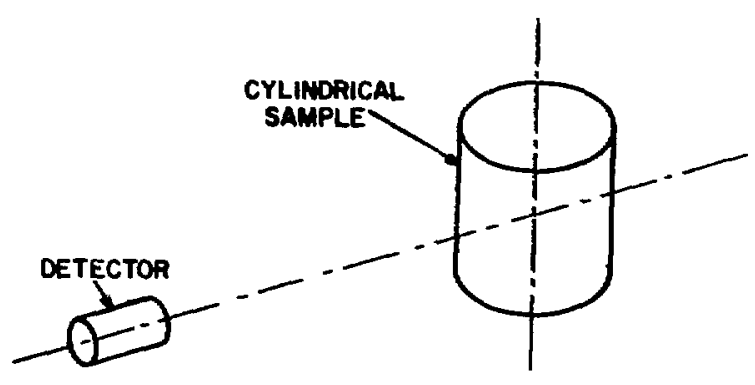

F2. 8. Typical asuay peometry for which a two-dimensional model for computing CF(AT) is usully adequate. 


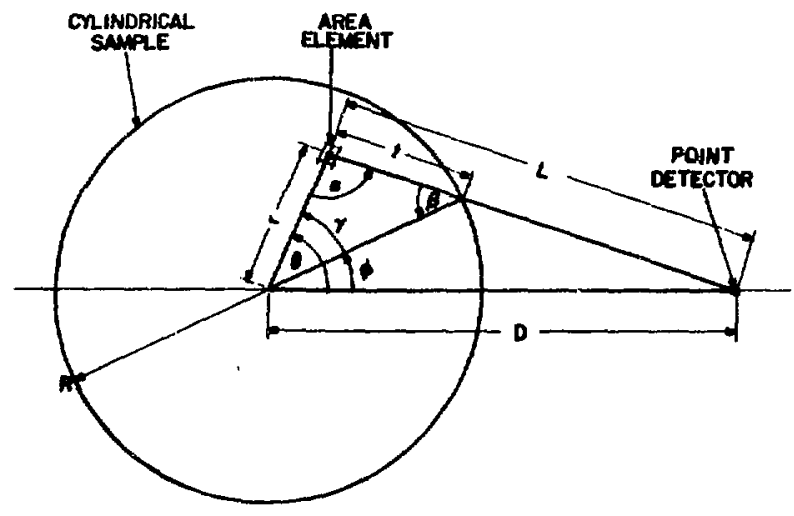

Fis. 9. Two-dimensional model for computing CF(AT) showing the distances that must be determined and the variables in terms of which they must be expressed. Note that $0 \leq \alpha \leq \pi, \beta \leq \pi / 2$, and $0 \leq \gamma \leq \pi$.

But $\gamma=\pi-\alpha-\beta$ so that if $\alpha$ and $\beta$ are found, $\gamma$ is determined. From the law of cosines,

$$
\alpha=\cos ^{-1}\left(\frac{\mathrm{L}^{2}+\mathrm{r}^{2}-\mathrm{D}^{2}}{2 \mathrm{rL}}\right) \text {, }
$$

and from the law of sines, we find

$$
\beta=\sin ^{-1}\left(\frac{r}{R} \sin \alpha\right),
$$

Expressed in integral form, the correction factor with respect to a nonattenuating cylinder is given according to the definition of Eq. (7) as

$$
C F(A T)=\frac{\int_{\theta=0}^{\pi} \int_{r=0}^{R} \frac{d A(r)}{L^{2}(R, D, r, \theta)}}{\int_{\theta=0}^{\pi} \int_{r=0}^{R} \frac{e^{-\mu^{\ell}(R, D, r, \theta)} d A(r)}{L^{2}(R, D, r, \theta)}},
$$

where the area element in cylindrical coordinates $\mathrm{dA}=$ r dr $\mathrm{d} \theta$ and where a uniform distribution of activity over the sample area is assumed. Because of the obvious symmetry, the integral needs to be performed only over half of the cylindrical sample. When $t$ and $L$ are given as explicit functions of $R, D, r$, and $\theta$ according to Eqs. (23)-(26), the double integral in the denominator becomes complex and cannot be integrated in terms of elementary functions. The numerator can be integrated, yielding the expression $-(\pi / 2) \ln \left(1-\mathbf{R}^{2} / D^{2}\right)$. Numeric methods must then be used to evaluate the denominator of the expression for CF(AT).

One method is outlined here. More sophisticated procedures no doubt exist. Let the radius of the cylinder $R$ be divided into $N$ intervals and let the whole $\pi$ radian range of $\theta$ be divided into $M$ intervals. The radial and angular increments are then given by

$$
\Delta \mathrm{r}=\frac{\mathbf{R}}{\mathbf{N}},
$$

and

$$
\Delta \theta=\frac{\pi}{\mathbf{M}}
$$

Let the coordinates of the area element $\triangle \mathrm{A}$ be given by

$$
r=\Delta r(n-0.5) \text { with } n=1, \ldots, N
$$

and

$$
\theta=\Delta \theta(m-0.5) \text { with } m=1, \ldots, M
$$

The area of the area element at $r$ and $\theta$ is then given by

$$
\begin{aligned}
\Delta A(n, m) & =\left\{\pi(n \Delta r)^{2}-\pi[(n-1) \Delta r]^{2}\right\}\left(\frac{\Delta \theta}{2 \pi}\right) \\
& =[(2 n-1) / 2](\Delta r)^{2}(\Delta \theta) .
\end{aligned}
$$

An approximation to the denominator of Eq. (27) can be written as a sum with $d A$ replaced by $\Delta A$, with $L$ and $t$ written in terms of $R, D, r$, and $\theta$ according to Eqs. (23)(26), and with $r$ and $\theta$ given in terms of the indices of summation $n$ and $m$ according to Eqs. (30) and (31). The correction factor with respect to the nonattenuating sample can then be written as

$$
C F(A T) \simeq \frac{\frac{\pi}{2} \ln \left(1-\frac{R^{2}}{D^{2}}\right)}{\sum_{m=1}^{M} \sum_{n=1}^{N} \frac{e^{-x^{2}(m, n)} \Delta A(n)}{L^{2}(m, n)}},
$$

It is often desirable to use the transmission $T$ of the sample as an input parameter to the computation in- 
stead of the linear attenuation ccefficient $\mu^{2}$, in which case $\mu^{2}$ is expressed as

$$
\mu^{2}=\frac{-\ln (T)}{2 R}
$$

If one is computing the $\mathrm{CF}(\mathrm{AT})$ with respect to a nonattenuating point at the center of the sample, the numerator in Eq. (27) integrates easily to $\pi R^{2} /\left(2 D^{2}\right)$ while the denominator remains the same. For the simple two-dimensional model, then, the ratio of the CF(AT) with respect to the nonattenuating sample to the CF(AT) with respect to a nonattenuating point at the center of the sample is $-\left(D^{2} / R^{2}\right) \ln \left(1-R^{2} / D^{2}\right)$, which depends nnly on the ratio $D / R$ and not on the value of $\mu^{\ell}$ (or of $\mathrm{T}$ ).

As mentioned before, it is often convenient to express or plot the values of $\mathrm{CF}(\mathrm{AT})$ as a function of the transmission $T$ rather than of the linear attenuation coefficient $\mu^{\ell}$. Plotting as a functon of $T$ is particularly convenient in presenting the results of this two-dimensional mociel because for a ñxed value of $T$, the $C F(A T)$ are a function only of the ratio $D / R$. This means, for example, that for a given value of $T$, a sample of $R=10$ $\mathrm{cm}$ and $\mathrm{D}=1000 \mathrm{~cm}$ has the same $C F(A T)$ as a sample of $R=100 \mathrm{~cm}$ and $D=10000 \mathrm{~cm}$. Figure 10 gives $\mathrm{CF}(\mathrm{AT})$ with respect to the nonattenuating sample as a function of $D / R$ for several values of $T$. The essential

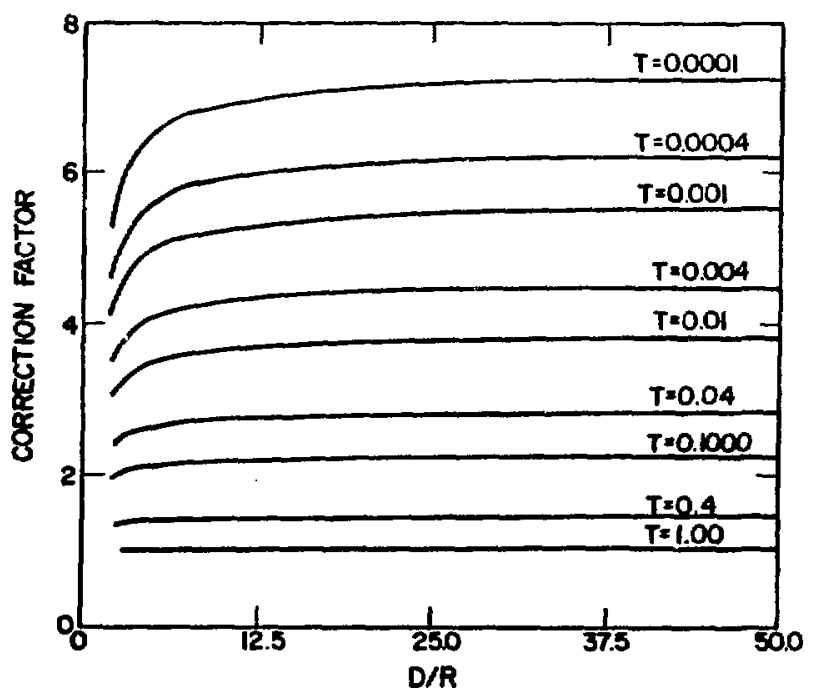

F2. 10. Correction factors with respect to a nonattenuating sample as computed from the two-dimentional model. They are pletted vs the mitio $D / R$ for various values of the transmission $T$, where $D$ is the distance from the center of the cylindrical sample to the point detector and $R$ is the radius of the sample. point is that the $C F(A T)$ decrease slowly as $D / R$ decreases; the larger changes occur for the smaller values of T. Remembering the definition of CF(AT), we can see qualitatively that this kehavior is a consequence of the inverse square law. It is clear that for a given value of $T$, CF(AT) asymptotically approaches a maximum as $D / R \rightarrow \infty$. To show quantitatively the deviations from the far-field $(D / R=\infty)$ case as $D / R$ decreases, the deviations are plotted in Fig. 11 as a function of $T$ for various values of $D / R$. For $T>0.001$ and $D / R>50$, all deviations are $\leq 1 \%$. Therefore, $D / R \geq 50$ can be regarded as the far-field situation for most purposes. It is also important to notice that the variation of CF(AT) as a function of $T$ is much stronger than the variation with the ratio $D / R$.

The results presented above were obtained with a minicomputer using values of $M=200$ and $N=200$ for which all the results are within $0.1 \%$ of what the actual integrals would give. The total number of area elements computed was obviously 40000 , and the time required was $\sim 2$ min per value. The exact time required for execution will obviously depend greatly on the computing equipment and programming language used. The point is that ior two-dimensional numeric integrations, results of high accuracy can be obtained in about a minute. The motivation for using a simple two-dimensional model when it is adequate is clear. If we go to a three-dimensional model, a modest extension in derivation and programming, and the third dimension is

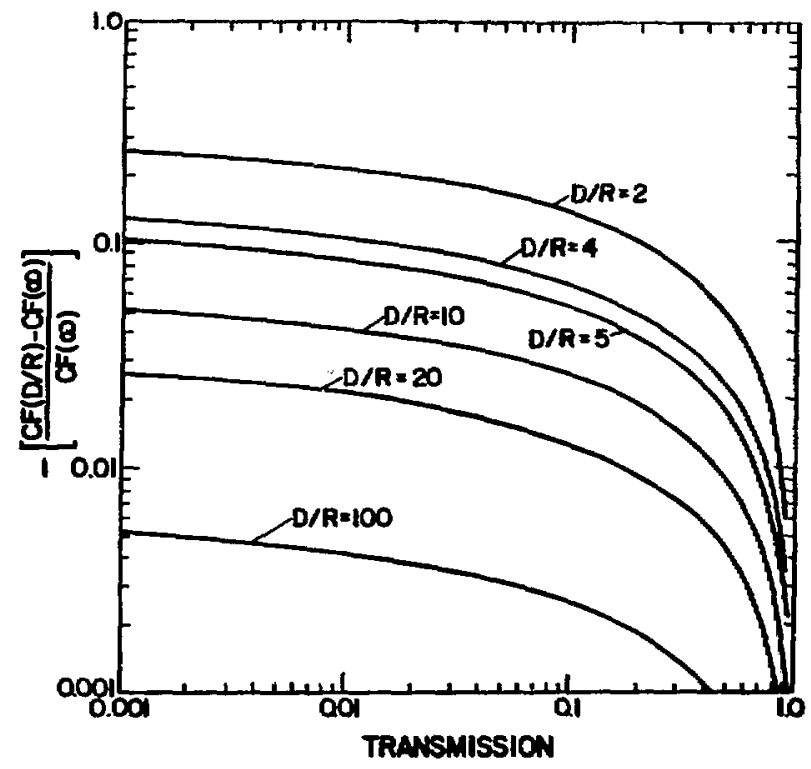

Fh. 11. Deviations of near-field values of CF(AT) from the frr-field values as a function of tranemizcion for various values of D/R. For $T$ $>0.001$ and $D / R \geq 50$, all deviations are $\leq 1.0 \mathrm{~m}$. Thus, $D / R \geq 50 \mathrm{cas}$ be repurded as "fir field" in muny situntions. 
also given 200 increments, the required execution time immediately increases to hundreds of minutes, a much more expensive and tedious proposition. Nevertheless, with judicious choices of the number of increments allotted to the three variables over which summing is done, three-dimensional numeric integration is feasible, though it is almost certain that an interpolation scheme will be needed to obtain values between those computed for selected values of $T$ or other parameters.

4. An Example of a Three-Dimensional Model. As a final example of an effort to construct a simple, yet adequate, model for an assay geometry, consider the segmented assay of cylindrical samples. In this case (Fig. 12), a detector views the sarnple through a horizontal collimator, which defines (with more or less overlap) segments, or slices, of the sample that are assayed individually. The sample is usually, but not always, as close to the collimator as possible. The detector is, of course, of finite size, often being a right circular cylinder of germanium $\sim 5.0 \mathrm{~cm}$ in diameter and $\sim 5.0 \mathrm{~cm}$ long. Experience has shown that for assay by the segmented procedure, the inverse square law effects caused by the collimator must be considered explicitly. It is generally not good enough to simply consider a very thin cylindrical, two-dimensional sample as described by the model in Sec. IV.C.3.

With respect to modeling the detector, the first thought might be to consider it as a point centered at the rear of the collimator. However, because some gamma rays are emitted in places within the sample where only a portion of the detector is seen through the collimator, the point detector assumption is not adequate. $A$ little thought suggests that a vertical line detector centered at the rear of the collimator might be an adequate model. The results of such an assumption are quite good and the expressions, which are derived below, will be based on it. Experimental results and more thought about the finite size of the actual detector suggest one further simple improvement in the model. Clearly, the gamma rays do not interact right at the rear of the collimator but

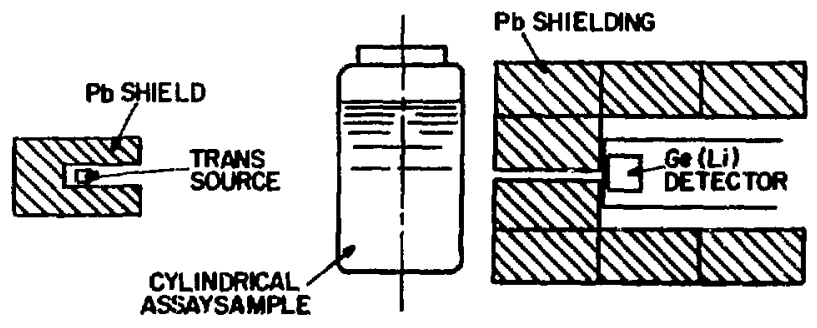

F2. 12. Typical segmented assay situation for which a three-dimensional model for computing $\mathrm{CF}(\mathrm{AT})$ is appropriate. actually behind the collimator in the volume of the detector, the distance from the rear of the collimator to the average interaction location being often as much as several centimeters. The results of the CF(AT) computations are improved a little by increasing the distance traveled by gamma rays to the line detector of the model by a constant length con?puted from the known average mean free path in the detector material for gamma rays of the energy concerned.

In summary, the model from which the required expressions will be derived consists of a perfect collimator (that is, one with no leakage) and a vertical line detector centered at the rear of the collimator. The efficiency of the "detector" is assumed to be independent of either the position or angle at which the gamma rays strike it, and for computation the distance from the emitting element to the line detector of the mathematical model will be increased by a constant approximately equal to the distance from the line detector to the average interaction lccation in the detector.

Though somewhat more complex than the first two examples, this model is still much simpler than the real geometry but provides accurate values of $C F(A T)$ over a wide range of transmission, especially with the addition of the penetration depth constant. Adequately accurate means generally within $\sim 1 \%$ of the correct values, which in most cases of the assay of solid samples, is satisfactory because the ultimate accuracy of the assay of such samples almost always depends more on the heterogeneities that so frequently exist within them than on the exact function used for CF(AT).

Inasmuch as materials are often packed in metal containers that significantly attenuate the emitted gamma rays, the packaging should be, in general, included in the model. As will be seen, this is straightforward. The necessary package parameters (inside diameter, outside diameter, and $\mu^{\mathbf{l}}$ ) are usually known with sufficient accuracy. In the case to be discussed below, the outside diameter of the sample may be different from the inside diameter of the container, a situation that arises quite often when double containment is used.

Qualitative considerations indicate that if cylindrical samples of different diameters are to be assayed in a common geometry by the segmented procedure or if the standards are of different diameters and/or packaging than the unknowns, the CF(AT) are best calculated with respect to a nonattenuating line source along the axis of the sample. By doing this, the corrected rates from samples of different diameters with different package materials and thicknesses can be directly compared, thus permitting the assay of unknowns with respect to standards of different diameters and packaging. $A$ line rather than a point is required as the simpler specified 
shape because the influence of the collimator must be included.

When the sample packaging is included in the model, more possibly useful definitions of $C F(A T)$ can be given. Although the CF(AT) with respect to the nonattenuating line is generally the most useful in segmented scanning, it is worthwhile to list some of the possible definitions of CF(AT) for the segmented geometry to emphasize the fact that there are several useful approaches. First, if the package is ignored, there are the two obvious definitions:

1. $C F(A T)$ for an attenuating cylindrical sample with respect to a norattenuating cylindrical sample. This is simplest in concept, and it is adequate if samples in identical packages of negligible attenuation are to be assayed.

2. CF(AT) for an attenuating cylindrical sample with respect to a nonattenuating line sarnple. If samples of varying diameters in packages of negligihle attenuation are to be assayed, this too is an adequate definition.

Now when samples in packaging of significant attenuation must be considered, we can write more derinitions; three of them are given below.

3. CF(AT) for an attenuating cylindrical sample and package with respect to a nonattenuating cylinder. This definition is applicable, for example, to samples in packages of the same dimensions but different materials and/or thickness.

4. $C F(A T)$ for an attenuating cylindrical sample and package with respect to a nonattenuating cylinder in an attenuating package. This would be suitable for identical samples of the same diameter in packaging of significant attenuation.

5. $\mathrm{CF}(\mathrm{AT})$ for an attenuating cylindrical sample and package with respect to a nonattenuating line sample. This is probably the most general and useful of any of these definitions, though it is not quite as simple as some of the others.

Mathematical expressions will be given for all five definitions. The main intent is to show that there are often several alternative definitions and that care should be taken to use one that is commensurate with the desired or possible accuracy of a given assay situation. After the model is set up and the basic trigonometry is done, it is just about as easy to compute according to one definition as another.

Figure 13 gives typical output from a code that gives results for all five definitions. All the parameters given in the upper part of Fig. 13 are input by the user except the $D / R$ ratio and maximum sample height seen by the
CODE CYLCL

NUTERTC INTEORATION FOR CONRECTION FACTORS FOR 3-D CYL WITH COLLIMATIOK. ASSUAES EBSENTIALLY LIMEAK DETECTON AND CONBIDERB FRACTIOH OF DETECTOR SEEN IM PENUHARA REBIOH. COHPUTES FIUE DIFFERENT CORRECTIDN FACTBRS.

MUHBER OF RADIAL INCREMEMTS

MUHER OF AHOULAR INCREMENTS IH PI RADIAWS: 50

MUHAER OF Z-INCREHENTS IM HALF HEIEHT

DISTANCE SAMPLE-CENTER TO DETECTOR(CH) = 17

BAMPLE RADTUS (CH)

RATIO OF D/R

CONTAINER IR (CH)

COHTAIMER OR (CH)

COLLIMATOR DEPTH (CM)

COLLIHATOR HEIOHT (CW)

CIS REAR OF CQLL TO IMTERACTION PT IM OET E

MAX REAR OF COLL TO INTERACTIOH PT IH DET

CONTAINER TRANSHTgSION

CF 1=CF FOR CYL URT MON ATT CY

CF2=CF FOR CYL UAT NON-ATT LINE

CF $3=$ CF FOR CYL + CONTAINER WRT NON-ATT CYL

CFA-CF FOR CYL + CONTAINER WRT CON-ATT LIME

CFS=CF FOR CYL + CONTAINER URT HON-ATT CYL ATT COMTAIMER

$\begin{array}{lllll}\text { TRAHS } & \text { MU(1/CM) } & \text { CF3 } & \text { CFA }\end{array}$

$.02 \quad .465717$

$3.38697 \quad 3.40347$

$\sin 20903$

2.00708

.225848

3.50473

3.52181

3.3954

1359773

2.01686

2.07469

2.0848

2.00997

1.6055

1.65074

1.65879

1.59925

DAY TIME AT START OF EXECHTIOHOA-AUD-82 14:44:30 DAY TIHE AT EMD OF EXECUTIOH O4-AUB-82 30:17:27

Fig. ij. Sample output from a code using the three-dimensional model for $\mathrm{CF}(\mathrm{AT})$ in segmented assays. Results are given for five different, sometimes useful, definitions of CF(AT).

detector, both of which are computed from the other parameters. Execution time will clearly be shorter if fewer definitions are computed, but it is a much stronger function of the equipment used. For example, the output of Fig. 13 was produced in 18660 s per transmission value with a BASIC code running on a DEC PDP 11/34 computer without hardware floating point arithmetic capability. The same results were obtained by a FORTRAN code running on a DEC PDP $11 / 60$ computer with hardware floating point arithmetic capability in only $283 \mathrm{~s}$ per transmission value, 66 times faster.

Finally, the expressions used in computing the values of CF(AT) for the five definitions will be derived. It will be seen that once the geometry and appropriate variables are defined, the task is basically an exercise in trigonometry. Figure 14 shows the modei' geometry from above, along with the pertinent parameters and variables. Figure 15 shows a vertical section of the assay geometry along line $L$ in Fig. 14. The task consists of expressing in terms of the parameters and the volume element coordinates the following quantities:

$\lambda=$ the distance of volume element to detector,

f $\quad$ the fraction of detector viewed from the volume element, 


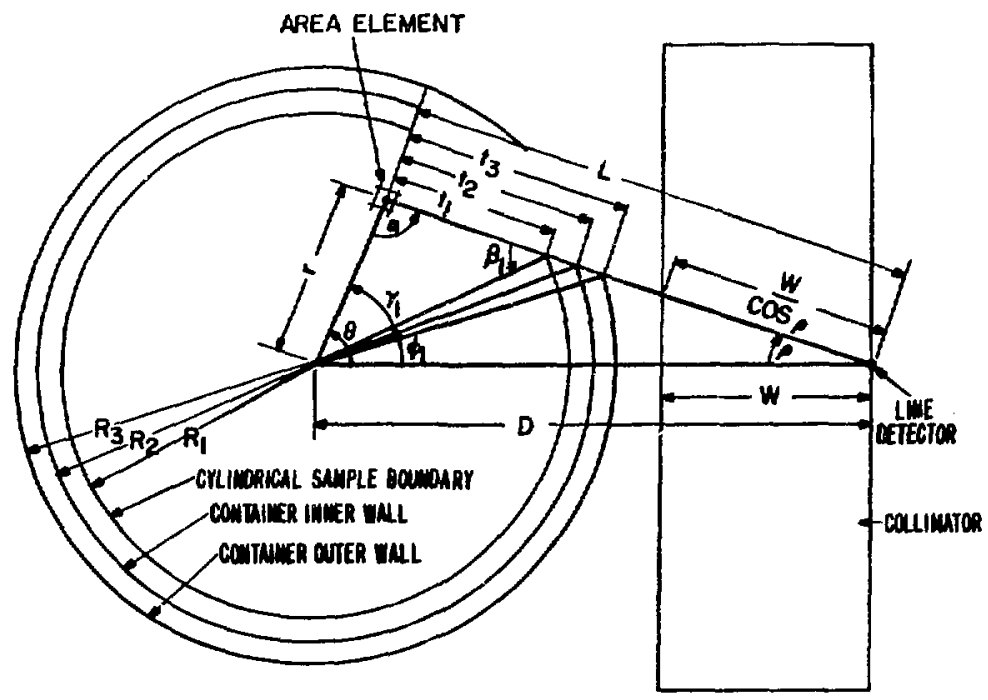

Fig. 14. Horizontal sectional view of the three-dimensional model for computing $\mathrm{CF}(\mathrm{AT})$ showing required distances and the variables in terms of which they must be expressed. Note 1 : angles $\theta_{2}, \theta_{3}, \gamma_{2}, \gamma_{3}, \beta_{2}$, and $\beta_{3}$ correspond in an obvious way to angles $\theta_{1}, \gamma_{1}$, and $\beta_{1}$. Note $2: 0$ $\leq \alpha \leq \pi, \beta \leq \pi / 2,0 \leq \gamma \leq \pi$, and $\rho<\pi / 2$.

$\tau_{1} \quad=$ the distance traveled by the gamma rays from the volume element within the sample,

$\tau_{3}-\tau_{2}=$ the distance traveled by the gamma rays from the volume element through the container walls, and

$\mathrm{F} \quad=$ the fraction of sample volume of a given volume element.

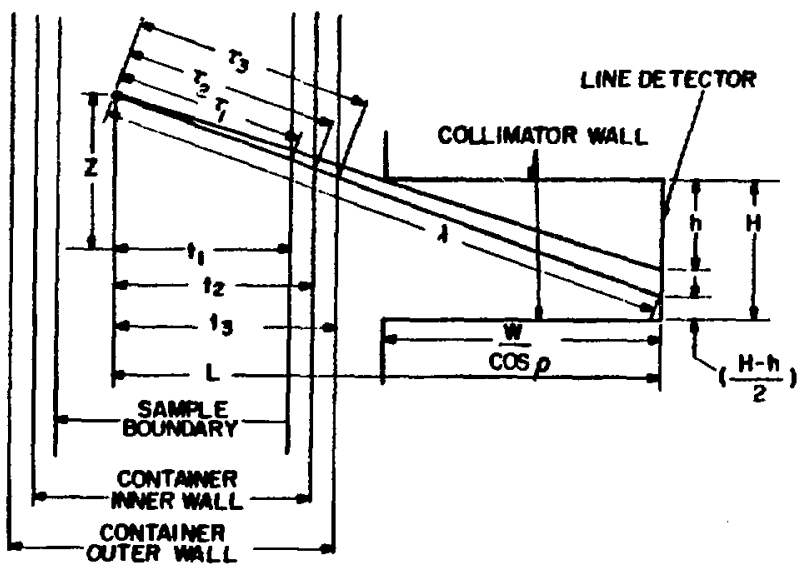

Fi. 15. Vertical sectional view of the three-dimensional model for computing $C F(A T)$ showing required distances and the variables in terms of which they must be expressed. The section is along line $L$ in Fig. 14.
We will adopt a cylindrical coordinate system with origin at the center of the cylindrical sample and with the z-axis along the axis of the sample. The coordinates of the volume elements will be $r, \theta$, and $z$. The projections of the required distances on the $r, \theta$ plane will be derived first, and then the expressions will be extended to the third dimension.

Referring to Fig. 14, the section in the horizontal plane, and using the law of cosines, we obtain

$$
L=\sqrt{D^{2}+r^{2}-2 D r \cos \theta},
$$

which is the total distance traveled in the horizontal plane by gamma rays from the volume element at $r, \theta, z$ to the detector. Next consider the distances $t_{1}, t_{2}$, and $t_{3}$, all of which are required to compute the total distances traveled by gamma rays on the way to the detector through the sample and its packaging. Various equivalent formulations are possible; the one given here is different from that for the corresponding distances in the two-dimensional case in Sec. IV.C.3. If the angles $\alpha$ and $\gamma_{1}$ of Fig. 14 are known, one can write by the law of sines

$$
t_{1}=R_{1} \frac{\sin \gamma_{1}}{\sin \alpha}
$$


From Fig. 14, it is seen that $\alpha=\pi-\theta-\rho$ and that by the law of sines again

$$
\rho=\sin ^{-1}\left(\frac{r}{L}\right) \sin \theta
$$

so that $\alpha$ is now fully determined. Again referring to Fig. 14 , we see that $\gamma_{1}=\pi-\alpha-\beta_{1}$, and by the law of sines yet again

$$
\beta_{1}=\sin ^{-1}\left(\frac{D}{R_{1}} \sin \rho\right)=\sin ^{-1}\left(\frac{D}{R_{1}} \frac{r}{L} \sin \theta\right)
$$

so that $\gamma_{1}$ is also fully determined. The expressions for $t_{2}$ and $t_{3}$ are identical to those for $t_{1}$ with the subscript 1 becoming 2 and 3, respectively. Referring again to Fig. $14, \rho<\pi / 2$ and $\beta_{1}<\pi / 2$ so that the $\sin ^{-1}$ function will give no trouble in writing codes.

Now referring to Fig. 15, let us find the actual distances traveled on the way to the detector by gamma rays from the volume element at $r, \theta, z$. Consider two cases, the first for $z \leq H / 2$ and the second for $z>H / 2$. For the $\mathrm{z} \leq \mathrm{H} / 2$ case, the whole detector is viewed. It is assumed that the efficiency is equal all along it and that the distance from the volume element to any point on the detector is the distance to the center of the detector. With these simplifying assumptions, we have

$$
\lambda=L \sqrt{1+z^{2} / L^{2}} .
$$

Letting $a=\sqrt{1+z^{2} / L^{2}}$, we have by similar triangles

$$
\tau_{1}=a t_{1}, \tau_{2}=a t_{2} \text {, and } \tau_{3}=a t_{3} \text {. }
$$

Letting $f$ be the fraction of the detector viewed, we have $f=1$ for $z \leq H / 2$.

For the $\mathrm{z}>\mathrm{H} / 2$ case, we try to take into account the fact that only part of the detector is viewed from a volume element at $r, \theta, z$. It is assumed that the collimator is perfect, that is, there is no leakage through the edges. It is additionally assumed that all the viewed portion of the detector is equally efficient, and that the distance traveled to the detector by all gamma rays is the distance to the center of the area viewed. With these simplifying assumptions, by similar triangles the unviewed detector length is

$$
h=\frac{(z-H / 2)}{(L-w / \cos \rho)}\left(\frac{w}{\cos \rho}\right) .
$$

For $h<H$, the distance from the volume element at $r, \theta, z$ to the center of the portion of the detector viewed is given using the Pythogorean Theorem and minor algebra by

$$
\lambda=L \sqrt{1+\frac{(z+h / 2)^{2}}{L^{2}}} .
$$

Letting $b=\sqrt{1+(z+h / 2)^{2} / L^{2}}$, using similar triangles we have

$$
\tau_{1}=b t_{1}, \tau_{2}=b t_{2} \text {, and } \tau_{3}=b t_{3} \text {. }
$$

Here the fraction of detector viewed is given by

$$
f=1-\frac{h}{\bar{H}} \text {. }
$$

One more geometrical relationship is needed, the length of cylindrical sample over which to do the numerical integration. We seek the value of $z$ for which the fraction of the detector viewed becomes zero for all values of $r$ and $\theta$; this value is clearly obtained for $r=R_{1}$ and $\theta=\pi$. Letting $Z$ equal the maximum value of $z$ and using similar triangles, we obtain

$$
\mathrm{Z}=\left(\mathrm{D}+\mathrm{R}_{\mathbf{1}}-\frac{\mathrm{W}}{2}\right)\left(\frac{\mathrm{H}}{\mathrm{W}}\right)
$$

Now it is desired to compute CF(AT) with respect to a nonattenuating line, that is, with the source obtained if the cylindrical sample and its container collapse radially into a nonattenuating line along the axis of the sample. The expressions for the distance to the detector will change somewhat, and again there are cases for $\mathrm{z} \leq \mathrm{H} / 2$ and for $z>H / 2$. Letting $\lambda_{z}$ be the distance from the line element at $r=0$ and $z$, we have for $z \leq H / 2$

$$
\lambda_{2}=\mathrm{D} \sqrt{1+\frac{\mathrm{z}^{2}}{\mathrm{D}^{2}}}
$$


As before, $f=1$ for $z \leq H / 2$. For $z>H / 2$, we have

$$
\lambda_{2}=\mathrm{D} \sqrt{1+\frac{\mathrm{z}+\frac{\mathrm{h}^{2}}{2}}{\mathrm{D}^{2}}},
$$

where

$$
h=\left(\frac{\mathrm{z}-\frac{\mathrm{H}}{2}}{\mathrm{D}-\mathrm{W}}\right) \mathrm{w} .
$$

For $\mathrm{z}>\mathrm{H} / 2$, as before, $\mathrm{f}=1-\mathrm{h} / \mathrm{H}$.

In performing the numeric integrations, let the range of the volume element coordinates $r, \theta, z$ be divided into $\mathbf{I}, \mathrm{J}, \mathrm{K}$ intervals, respectively, where $\mathrm{r} \leq \mathbf{R}_{1}, 0 \leq \theta \leq \pi$, and $0 \leq z \leq Z$. The increments in $r, \theta, z$ are then

$$
\Delta \mathrm{r}=\mathrm{R} / \mathrm{I}, \Delta \theta=\pi / \mathrm{J}, \text { and } \Delta \mathrm{z}=\mathrm{Z} / \mathrm{K}
$$

Letting the indices of summation corresponding to $r, \theta, z$ be $i, j, k$, respectively, the coordinates of the volume element are

$$
\begin{aligned}
& r=\Delta r(i-0.5) \text { for } i=1, \ldots, I \\
& \theta=\Delta \theta(j-0.5) \text { for } j=1, \ldots, J \\
& z=\Delta z(k-0.5) \text { for } k=1, \ldots, K
\end{aligned}
$$

In terms of $i, j, k$ and $\Delta r, \Delta \theta, \Delta z$, the volume element is given by

$$
\Delta V(i)=\frac{(2 i-1)}{2}(\Delta r)^{2}(\Delta \theta)(\Delta z),
$$

and its fraction of the volume of a cylinder of radius $R$ and height $2 Z$ is given by

$$
F(i)=(2 i-1)\left(\frac{\Delta r}{R_{1}}\right)^{2}\left(\frac{\Delta \theta}{2 \pi}\right)\left(\frac{\Delta z}{2 Z}\right) .
$$

For summing the nonattenuating line source, the sum is obviously only over $k$, and the volume element becomes just the line element $\Delta \mathrm{z}=\mathrm{Z} / \mathrm{K}$. The fraction of the total "volume" in $\Delta z$ is $F_{2}=1 / 2 \mathrm{~K}$ because the total length of the line is $2 Z$.
Either $\Delta V(i)$ or $F(i)$ may be used in the summations of the numeric integrations because the expressions for CF(AT) are ratios of sums. Using $F(i)$ seems prezerable because it is independent of the units of length used.

As in the two-dimensional case, the computations can clearly be done with specific values of the linear attenuation coefficient for the sample $\mu^{l}$ and the container $\mu_{c}^{l}$ or alternatively for corresponding values of transmission. Because the transmission is usually the measured parameter, it is preferred to use it as input to the computational codes. We have then

$$
\mu^{\prime}=\frac{-\ln (\mathrm{T})}{2 \mathrm{R}_{1}},
$$

and

$$
\mu_{c}^{q}=\frac{\ln \left(T_{c}\right)}{\left[2\left(R_{3}-R_{2}\right)\right]}
$$

If we take advantage of the symmetrical geometry, the numeric integration needs to be done only over onefourth of the cylindrical volume, and the range of $\theta$ and $z$ have been chosen accordingly. However, it is important to remember that $F$ is the fraction of the total volume, so one must properly normalize when comparing a volume sum with a line sum.

To compute the five different correction factors desired in this illustrative example, five sums must be formed from the expressions given above. The expressions for $F, f$, and $\lambda$ are given in terms of the geometrical parameters and the coordinates $r, \theta, z$ according to the equations above. In calculating the sums, one must remember that the forms of $f, \lambda, \tau_{1}, \tau_{2}$, and $\tau_{3}$ are different for $\mathrm{z} \leq \mathrm{H} / 2$ and for $\mathrm{z}>\mathrm{H} / 2$. The sums are

$$
S 1=4 \sum_{i=1}^{I} \sum_{j=1}^{J} \sum_{k=1}^{K} \frac{F f}{\lambda^{2}},
$$

which is proportional to the count rate from a nonattenuating cylindrical sample without a container;

$$
S 2=4 \sum_{i=1}^{I} \sum_{j=1}^{J} \sum_{k=1}^{K} \frac{F f e^{-k^{2} \tau_{1}}}{\lambda^{2}}
$$

which is proportional to the count rate from an attenuating cylinder without a container; 


$$
S 3=4 \sum_{j=1}^{I} \sum_{j=1}^{J} \sum_{k=1}^{K} \frac{F f \exp \left[-\left[\mu^{\ell_{1}}+\mu_{c}^{\ell}\left(\tau_{3}-\tau_{2}\right)\right]\right\}}{\lambda^{2}},
$$

which is proportional to the count rate from an attenuating cylindrical sample with its attenuating container;

$$
S 3=4 \sum_{i=1}^{I} \sum_{j=1}^{J} \sum_{k=1}^{K} \frac{F f e^{-\left[\mu_{c}^{R}\left(\tau_{3}-\tau_{2}\right)\right]}}{\lambda^{2}},
$$

which is proportional to the count rate from a nonattenuating cylindrical sample and its attenuating container; and

$$
S 5=2 \sum_{i=1}^{K} \frac{F_{l} f}{\lambda_{c}^{2}},
$$

which is proportional to the count rate from a nonattenuating line source.

The factors 4 and 2 in the sums normalize so that each sum represents the count rate from the whole sample. Remember that $f=0$ for a portion of the volume of summation, a cylinder whose height is equal to twice the maximum $z$ for which any fraction of the detector is seen from the cylindrical sample. Also, at the extremes of the line source, the detector cannot be seen at all. Any values of $f$ that are negative when computed with the expressions above should be set to zero.

With the sums as given above, the expressions for the five correction-factor definitions previously listed are

$$
\mathrm{CF} 1=\frac{\mathrm{S} 1}{\mathrm{~S} 2}
$$

the $\mathrm{CF}(\mathrm{AT})$ for an attenuating cylindrical sample with respect to a nonattenuating cylindrical sample;

$$
\mathrm{CF} 2=\frac{\mathrm{S} 5}{\mathrm{~S} 2}
$$

the $\mathrm{CF}(\mathrm{AT})$ for an attenuating cylindrical sample with respect to a nonattenuating line sample;

$$
\mathrm{CF} 3=\frac{\mathrm{S} 1}{\mathrm{S3}}
$$

the $\mathrm{CF}(\mathrm{AT})$ for an attenuating cylindrical sample and container with respect to a nonattenuating cylindrical sample;

$$
\mathrm{CF} 4=\frac{\mathrm{S} 4}{\mathrm{S3}}
$$

the $\mathrm{CF}(\mathrm{AT})$ for an attenuating cylindrical sample and container with respect to a nonattenuating cylindrical sample and its attenuating container; and

$$
\mathrm{CF} 5=\frac{\mathrm{S} 5}{\mathrm{~S} 3}
$$

the CF(AT) for an attenuating cylindrical sample in an attenuating container with respect to a nonattenuating line sample.

5. Approximate Forms and Interpolation. In general, the most accurate way to compute CF(AT) for reasonable assay geometries is by the use of a relatively simple mathematical model and numeric integration as discussed above. However, as also explained before, because of the often lengthy execution times to compute CF(AT) by those procedures, it is often desirable for a given assay system and class of samples to compute $\mathrm{CF}(\mathrm{AT})$ for a few values of $\mathrm{T}$ (or $\mu^{\mathrm{N}}$ ) and to use an interpolation scheme to find $C F(A T)$ for intermediate values of $T$. This procedure becomes necessary rather than desirable if the particular assay system does not have adequate computing capability. The interpolation problem can clearly be approached in several ways; a few will be discussed below.

The brute force approach is to do point-to-point interpolations between enough computed values of CF(AT) to reduce the error in any interpolated value to the maximum acceptable level. This will surely work, but if it is to work in an automated way, the assay system computer must be given a table of CF(AT) values and an interpolation code. The scheme is quite adequate if sufficient computer capability is available and relatively few kinds of samples are to be assayed so that only a limited number of tables need to be provided. 
Since $C F(A T)$ has in general an approximate $\log (T)$ dependence (see Fig. 3), it is reasonable to fit to a set of values a function of the form

$$
\mathrm{CF}(\mathrm{AT})=\mathrm{A}+\mathrm{B} \log (\mathrm{T})+\mathrm{C}[\log (\mathrm{T})]^{2} .
$$

The assay system computer, whatever its capability may be, then need only store the constants $A, B$, and $C$ for each set of $C F(A T)$ to be fit. Experience has shown that this scheme works very well over quite wide ranges of T. For example, in a typical segmented scanning situation, $A, B$, and $C$ can be determined to give values of $C F(A T)$ correct to $\leq 0.3 \%$ for $0.008 \leq \mathrm{T} \leq 0.30$.

A particularly simple scheme, especially useful when the greatest accuracy is not required, is based on the farfield form for the CF(AT) for a slab, -ln (T)/ $(1-T)$. Observing that a circle is not after all so very different from a square and remembering that the $\mathrm{CF}(\mathrm{AT})$ for a thin cylindrical sample is a little less than that for a thin square sample whose side is the same as the cylinder diameter (see Fig. 3), one is led to try

$$
C F(A T) \simeq \frac{-\ln \left(T^{k}\right)}{\left(1-T^{k}\right)}
$$

with $\mathbf{k}<1$ as an approximate function for cylindrical samples, even in the near-field situation. This form also has $a \ln (T)$ dependence for $T \ll 1$ and has the virtue of being simple with only one constant to be determined. It has been used often, particularly a few years ago when more was dune with hand-held calculators or programmable desk calculators of limited capability than at present. Figures 16 and 17 provide a feeling for how accurate and useful the approximate form might be.
Figure 16 gives the fractional deviation of $-\ln \left(T^{*}\right) /$ $\left(1-T^{*}\right)$ from the correct far-field values for a cylinder [Eq. (15)] as a function of transmission for various values of $k$. Figure 17 is similar, but the comparison is for a near-field assay of a cylindrical sample where $(D / R)=5 / 1$ (distance from center of sample to detector five times sample radius). In Figure $16, k=0.82$ gives CF(AT) correct within $\pm 1 \%$ for $0.01 \leq T$ $\leq 1.0$, and in Fig. 17, $k=0.75$ gives $C F(A T)$ correct within $\pm 1.5 \%$ for $0.01 \leq T \leq 1.0$. However, as will be pointed out in the next section, the choice of $k$ that gives the smallest overall absolute error for CF(AT) may not be the best choice for assays in a particular range of $T$.

As a final example of an approximate function for CF(AT), the simplest and crudest of all, consider the function

$$
\mathrm{CF}(\mathrm{AT}) \simeq \sqrt{\mathrm{T}}
$$

For $T>0.2$, this function gives values $\leq 10 \%$ greater than $-\ln (T) /(1-T)$ (Table II). As crude as it is relative to other functions, $1 / \sqrt{T}$ is useful for rough work, particularly with low-resolution detectors. Notice that $1 / \sqrt{\mathrm{T}}$ implies that all the gamma rays are emitted at the center of the sample.

The major point of this discussion is that various interpolation procedures and approximate functions for CF(AT) are available. Just what is done will depend on the accuracy desired or possible for a given class of materials to be assayed. If, for example, the problem is a field measurement of a heterogeneous drum containing ${ }^{235} U$, the accuracy will probably be determined far more by the degree of heterogeneity of the sample material than by the function used for CF(AT). In such a case, where $\pm 25 \%$ accuracy may be all that can be hoped for,

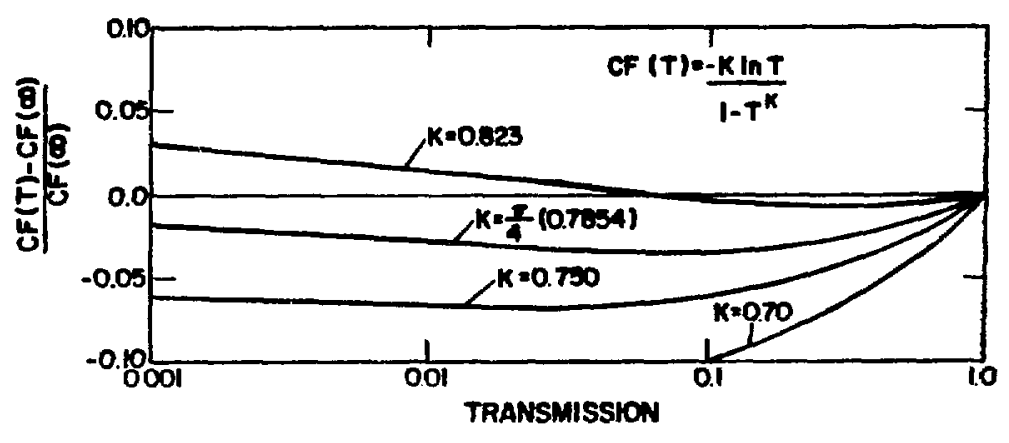

Fis. 16. Deviations of the values of CF(AT) computed from the approximate expreasion $C F(T)=-k \ln (T) /\left(1-T^{h}\right)$ from the far-field values for a cylinder. They are ploted as functions of the transmission $T$ for several values of the parameter $k$. 


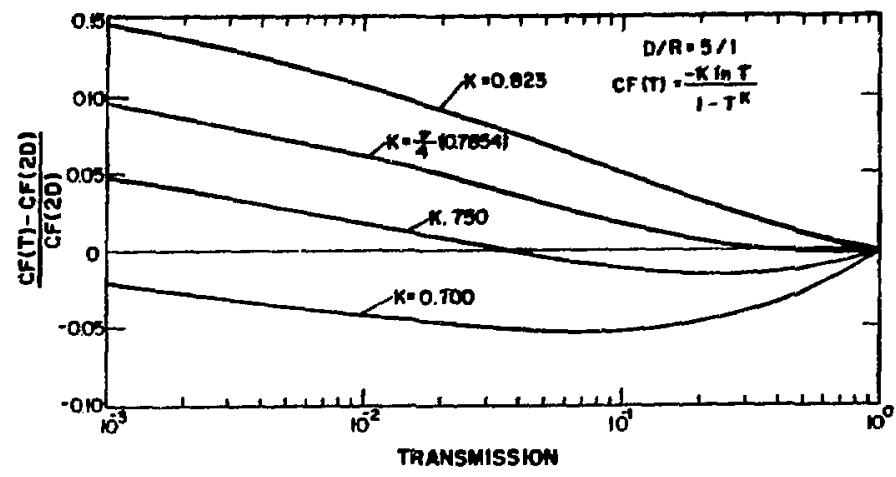

Fis. 17. Deviations of the values of $C F(A T)$ computed from the approximate expression $C F(T)=-k$ In $(T) /\left(1-T^{k}\right)$ from the values from the twodimensional model for cylindrical samples for. $D / R=5$. They are plotted as functions of the transmission $T$ for several values of the parameter $k$.

the work of setting up a model and doing numeric integrations for $\mathrm{CF}(\mathrm{AT})$ is wasteful. On the other hand, if the samples are solutions, which by definition meet the assumptions on uniformity, careful modeling and computation can result in assay results with accuracies $<1 \%$, and the effort is fully justified.

6. The Effects of Absolute and Relative Error in CF(AT). Throughout this report it is assumed that gamma-ray assay systems will be calibrated by use of suitable physical standards containing known amounts of the isotope being assayed. It is also assumed that CF(AT) will have to be determined for both the unknowns and the standards. Generally, the CF(AT) will be mainly a function of the measured transmission $T$ with some influence from the geometrical parameters, which may or may not be the same for both unknowns and standards.

The consequences of using a function for CF(AT), which is incorrect to some degree, should be in-

Table II. Comparison of Far-Field Correction Factor for Slab with Approximate Form $1 / V T$ as Function of Transmission ${ }^{2}$

\begin{tabular}{|c|c|c|}
\hline $\mathbf{T}$ & $1 / \sqrt{T}$ & $-\ln (T) /(1-T)$ \\
\hline $\begin{array}{l}1.0 \\
0.8 \\
0.6 \\
0.4 \\
0.2 \\
0.1\end{array}$ & $\begin{array}{l}1.000 \\
1.118 \\
1.291 \\
1.581 \\
2.236 \\
3.162\end{array}$ & $\begin{array}{l}1.000 \\
1.116 \\
1.277 \\
1.527 \\
2.012 \\
2.558\end{array}$ \\
\hline
\end{tabular}

vestigated because of the important influence the ac curacy of CF(AT) has on the accuracy of the final assay results. To do so, consider a situation as indicated in Fig. 18 in which a true and a false CF(AT) function are shown as functions of $T$, along with indication of different transmissions for the unknown and standard. Let the following notation be adopted for this demonstration:

$$
\begin{aligned}
& T=\text { transmission, } \\
& \mathbf{C F}(\mathrm{T})=\mathrm{CF}(\mathrm{AT}) \text { as a function of } \mathrm{T}, \\
& \mathbf{R R}=\text { raw rate for assay gamma ray, } \\
& \mathbf{T C R}=\text { total corrected rate for assay gamma ray, } \\
& \mathbf{K} \quad=\text { calibration constant, } \\
& \mathbf{G} \quad=\text { mass of assay isotope in unknown, and } \\
& \mathbf{M} \quad=\text { known mass of assay isotope in standard. }
\end{aligned}
$$

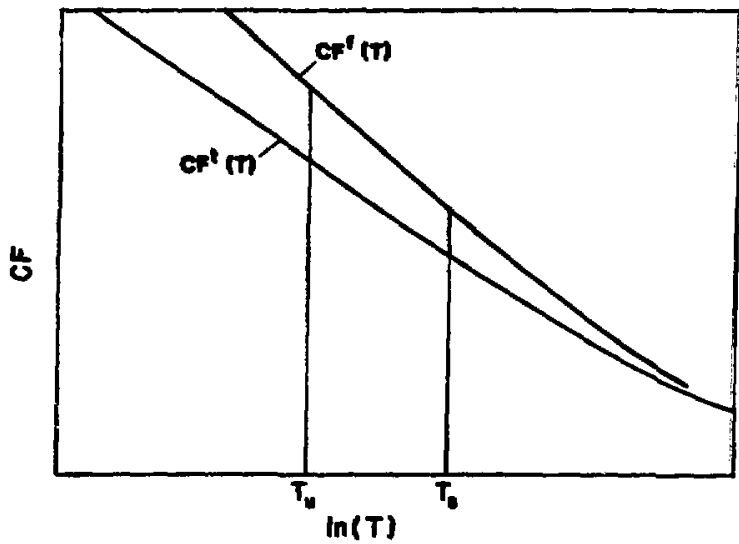

F3. 18. A hypothetical situation to illustrate the consequences of using an incorrect function for CF(AT). CF(T) repreaents the incorrect or fale function for CF(AT) as a function of transmission $T$ and $C F(T)$ represents the correct or true function for CF(AT). $T_{u}$ and $T_{3}$ represent the transmissions of unknown and standard, respectively. 
The superscripts $f$ and $t$ will be used to indicate quan. tities associated with the false and true functions for $C F(A T)$, respectively, and the subscripts $u$ and $s$ will similarly be used to indicate quantities associated with the unknown to be assayed and the standard used to calibrate. From Eq. (2), we can write

$$
\mathrm{G}=\frac{\mathrm{TCR}_{\mathrm{u}}}{\mathrm{K}}
$$

where from Eq. (1), ignoring $C F(R L)$,

$$
\mathbf{T C R}=\mathbf{R R} * \mathrm{CF}(\mathrm{T})
$$

and

$$
\mathbf{K}=\frac{\mathbf{T C R}_{\mathbf{s}}}{\mathbf{M}}
$$

As indicated in Fig. 18, we assume that the ratio of $\mathrm{CF}^{\mathrm{f}}$ to $C^{\prime}(T)$ is a well-behaved function of $T$ :

$$
\frac{C F^{f}(T)}{C F^{\prime}(T)}=C(T)
$$

Using the incorrect function for the CF(AT), we would determine an incorrect calibration constant as

$$
K^{f}=\frac{R R_{s} * C F^{f}\left(T_{s}\right)}{M}=\frac{R R_{s} * C F^{t}\left(T_{s}\right) * C\left(T_{s}\right)}{M},
$$

whereas the correct result should be

$$
K^{\mathfrak{t}}=\frac{R_{\mathbf{s}} * \mathrm{CF}^{\mathbf{t}}\left(\mathrm{T}_{\mathbf{s}}\right)}{\mathbf{M}}
$$

The TCR for the unknown will also be incorrectly determined as

$$
T_{C R}^{f}=R_{u} * \operatorname{CF}^{\prime}\left(T_{u}\right)=R_{u} * \mathrm{CF}^{\prime}\left(T_{v}\right) * C\left(T_{u}\right)
$$

whereas it should be

$$
\mathbf{T C R}_{\mathrm{u}}^{\prime}=\mathbf{R R}_{u} * \mathrm{CF}^{\prime}\left(\mathbf{T}_{\mathrm{u}}\right)
$$

Then the incorrect assay value will be determined as

$$
G^{f}=\frac{T_{C R}^{f}}{K^{f}}=\frac{2 R_{u} * C F^{\prime}\left(T_{u}\right) * C\left(T_{u}\right)}{R R_{s} * C F^{t}\left(T_{s}\right) * C\left(T_{s}\right)} * M
$$

whereas the correct answer is given by

$$
G^{t}=\frac{T_{C R}^{1}}{K^{t}}=\frac{R_{u} * C F^{t}\left(T_{u}\right)}{R R_{s} * C F\left(T_{s}\right)} * M
$$

The ratio of the two results then becomes

$$
\frac{G^{f}}{\bar{G}^{t}}=\frac{C\left(T_{u}\right)}{C\left(T_{s}\right)}=\frac{C F^{f}\left(T_{u}\right) / C F^{t}\left(T_{u}\right)}{C F^{f}\left(T_{s}\right) / C F^{1}\left(T_{s}\right)}
$$

It is now seen that the ratio of false to true results does not depend directly on the absolute error in CF(T) but only on a ratio of ratios, namely, the ratio of $\mathrm{CF}^{\mathrm{f}}$ to $\mathrm{CF}^{\mathrm{t}}$ at $T_{v}$ divided by the ratio of $\mathrm{CF}^{f}$ to $C F$ at $T_{3}$. If this ratio of ratios is $\sim 1.00$ over the range of $T$ among both standards and unknowns, the assays will be correct in spite of any absolute error in CF(AT).

This result demonstrates and emphasizes that it is easier to correctly calibrate an assay system for a narrow range of transmission (which usually also implies a narrow range of concentration of the assay isotope) than for a broad range. It also emphasizes why great care must be used in modeling the assay geometry and computing $\mathrm{CF}(\mathrm{AT})$ if high accuracy is required over a wide range of concentrations. Aithough determination 
of the correction factor for the rate-related losses resulting from deadtime and pileup, $C F(R L)$, is not the subject of this report, it is clear that a wide range of assay isotope concentrations often means a wide range of count rates, which in turn implies large variations in $C F(R L)$ and the consequent necessity for carefully determining it.

One might ask, considering the difficulty in computing a $C F(A T)$ as a function of $T$ that is correct over a wide range of $T$, why standards should not be used to determine a variable calibration constant as a function of $\mathrm{T}$ or perhaps of the gross count rate if that seems to be causing most of the problem. Indeed that can be done, but preferably only as a fine tuning of a system calibration. Remember that by so doing we are, as it were, sweeping under the rug the things we do not understand about the physics of the assay arrangement or are perhaps incorporating a maladjustment of the equipment into the system calibration. Surely all efforts should first be made to understand the physics, to have functions for $\mathrm{CF}(\mathrm{AT})$ and $\mathrm{CF}(\mathrm{RL})$ that are as correct as possible, and to make sure the equipment is working correctly before attempting to calibrate away the residual systematic errors.

7. Considerations of the Precision of CF(AT) and of TCR. It is important in gamma-ray NDA (and indeed most forms of NDA) to be able to estimate the precision of the assay. Precision as used here means essentially just repeatability, and a measurement result may be very precise but nevertheless very inaccurate. This is only a reminder that precision is not a guarantee of accuracy. Nevertheless it is still important in gammaray NDA to have a reasonable estimate of precision because it puts a minimum bound on the uncertainty that must be assigned to a measurement and allows the variation of subsequent measurements to be understood.

In a properly operating gamma-ray NDA system using modern electronics, the precision will be almosi totally a function of the random nature of the emission of the gamma rays being counted. The influence of electronic fluctuations and drifts in the equipment should almost never influence the precision of the NDA results at a level of $>0.1 \%$. The dominant statistical component of the precision of the assays can usually be estimated from the rull-energy peak areas that were the input data for the assay result and from their precisions. The overall precision, including any contribution from the equipment, is estimated from replicate assays, and this overall precision compared with that estimated from peak areas and their precisions usually forms the basis for evaluating the stability of the assay system, both electronically and mechanicaliy.
Herein the concern is only with the statistical precision of the assay and primarily with the influence of the precision of CF(AT) on the precision of the final assay results. As formulated in this report, the assay result-the computed mass of the isotope being assayed-is proportional to the total corrected rate TCR, which is given [see Eq.(4)] as

$$
\mathrm{TCR}=\mathrm{FEIR} * \mathrm{CF}(\mathrm{AT}) \text {, }
$$

where FEIR is, as usual, the full-energy interaction rate. Letting $\sigma()$ indicate the estimate of the standard deviation of the indicated quantity, $v$ (TCR) must be found for the assays. We will not describe the procedures used to derive the expressions for $\sigma(\mathrm{TCR})$, $\sigma($ FEIR), or the $\sigma[C F(A T)]$, which are covered in detail in many papers, reports, and texts (two relatively simple sources are Refs. 16 and 17). The intent here is only to emphasize a few points relative to obtaining a reasonable expression for estimating o(TCR).

The first point is that if TCR can be written as an analytic function of the peak areas that went into computing it, then an expression for o(TCR) can usually be derived in terms of those peak areas and their precisions. However, when $C F(A T)$ is found by the numeric procedures described above, for the sake of reducing bias and thus improving accuracy, TCR is not computed from an analytic function, and the question of how to compute $\sigma(T C R)$ remains. A useful approach to the problem, and the point most emphasized in this section, is to use an approximate analytic function for CF(AT) in formulating the expression for TCR from which the expression for $\sigma(T C R)$ is derived. If used in computing TCR, the approximate forms for CF(AT) are often not sufficiently accurate (that is why the numeric integration procedures are used in the first place), but if chosen reasonably well, they usually provide an adequately accurate expression for $\sigma(T C R)$. For example, in Sec. IV.C.1 a one-dimensional model was used to obtain by numeric integration values of $C F(A T)$ for the assay of cylindrical samples viewed normal to the cylinder face by a germanium detector. It is likely that for deriving an expression for o(TCR), one could use $C F(A T)=-\ln (T) /(1-T)$, where $T$ is the transmission through the face of the cylindrical sample. If that is not satisfactory, the modified form $C F(A T)=-\ln \left(T^{*}\right)$ ) $\left(1-T^{k}\right)$ with $0.5<k<1.0$ would almost surely be adequate. The proper value of $k$ would be chosen by comparison with precisions computed from replicate assays. By using the procedure just described, one can have the accuracy provided by numeric integration of a more accurate model for $\mathrm{CF}(\mathrm{AT})$ and still have good estimates of $\sigma(T C R)$. Most modern assay systems have enough computational power to do the extra computation with modest extra execution time. 
Note that although $\sigma(T C R)$ is the assay precision and is therefore what is usually required, $\sigma[C F(A T)]$ alone is also sometimes of interest. The expression for $\sigma[C F(A T)]$ will always be simpler than the one for $\sigma(T C R)$. If no iull-energy peak areas are common to the expressions for FEIR and $\mathrm{CF}(\mathrm{AT})$, then

$$
\sigma_{\mathrm{r}}(\mathrm{TCR})=\sqrt{\sigma_{\mathrm{r}}^{2}(\mathrm{FEIR})+\sigma_{\mathrm{r}}^{2}[\mathrm{CF}(\mathrm{AT})]},
$$

where $\sigma_{\mathrm{r}}(\mathrm{x}) \equiv \sigma(\mathrm{x}) / \mathrm{x}$, so that the expression for $\sigma[\mathrm{CF}(\mathrm{AT})]$ becomes explicitly part of the expression for $\sigma_{\mathrm{r}}$ (TCR). If there are peak areas common to the expressions for FEIR and CF(AT), the separation in Eq. (80) does not exist, and the expression for TCR must be written as an explicit function of the peak areas concerned. The expression for $\sigma(T C R)$ must be derived from this new function according to procedures described in the references, and the expression for $\sigma[\mathrm{CF}(\mathrm{AT})]$ generally will not be a recognizable part of it. Such expressions for precision will frequently be very complex, but considerable simplification can usually be achieved by judicious approximations in setting up the original expression for TCR and by dropping from the final precision expression terms that make insignificant contributions. The effort to make such simplifications is worthwhile in terms of both reduced computation time and better understanding of the main sources of imprecision in a particular assay procedure.

\section{REVIEW OF FACTORS GOVERNING THE NUMBER OF STANDARDS USED}

It is stated in the introduction that the objective of this report is to explain procedures whereby calibration standards can be appropriate without being particularly similar, either physically or chemically, to the unknowns being assayed. Indeed, the bulk of all the preceding material has been devoted to that goal. The current insistence that the range of the masses of the isotopes of interest in the standards used for a given NDA procedure span the expected range in the unknowns to be assayed can also be considerably relaxed. Although the evidence for this allegation is implicit in the forgoing sections, no explicit discussion has been given of how far and how safely a calibration may be extrapolated beyond the range of masses in the standards used. It is appropriate to end this report with a brief discussion of those matters.

The insistence that the standards span the range of expected masses to be assayed seems rooted in the expectation that a calibration function will be fitted to a plot of assay system response. When gamma-ray NDA systems are considered, that response might be assumed to be the RR of Eq. (2) or the FEIR of Eq. (3). Such plots will almost inevitably be quite nonlinear, and extrapolation of a fitted nonlinear function beyond the data points to which it is fitted is not particularly safe. However, as explained above, if the multiplicative correction factors $C F(R L)$ and $C F(A T)$ are properly defined and computed, a total corrected rate is obtained: $T C R=$ $R R * C F(R L) * C F(A T)$. This is the data acquisition rate tinat would be observed in the absence of electronic losses and if the sample were changed to a specified nonattenuating simple shape. If the CF(AT) for all samples assayed are computed with respect to the same specified simple shape, one arrives at Eq. (2), $T C R=K$ * $M$, where $M$ is the mass of isotope being assayed and $K$ is the calibration constant. The important point is that the calibration function is now linear, in fact, linear with TCR $=0$ when $M=0$ and is thus determined by one parameter, K. Extrapolation with such functions is far less hazardous than with the nonlinear calibration functions generally assumed in the past, and hence most of the logical force is taken from the requirement to have the mass range of the standards span that of the unknowns.

With Eq. (2) as a calibration function, one can in principle determine $K$, the calibration constant, with a single standard. However, in practice, it is wise to use several standards, spanning a reasonable range of mass of the assay isotope, and perhaps with variations in other parameters such as matrix density and composition or sample size. The object in using several standards is obviously to confirm that $C F(R L)$ and $C F(A T)$ are being correctly determined so that $T C R=K * M$ is independent of variations in $\mathbf{M}$, chemical composition, shape, and so forth. It must be emphasized that if some nonlinearity is detected, the first concern should be to correct the problem(s), whether in equipment or in computation of CF(RL) or CF(AT), so that the calibration is linear, rather than to proceed immediately to add terms to the calibration equation. To prematurely start modifying the calibration equation often simply disguises the effects of poorly set up equipment, incorrect algorithms for $C F(R L)$ and $C F(A T)$, equipment malfunction, or even cf outright ignorance of proper procedures and methods. After the equipment is functioning at optimal levels and the computational algorithms are as good as can be reasonably derived, if there is still some nonlinearity, then consideration can be given to modifying the calibration equation. Indeed, if the assays are to be made over a very wide range of isotope masses and/or count rates and very high accuracy is required, there may be residual nonlinearities greater than acceptable. Because such problems will often result from a wide range of count rates, which results from a wide range of assay isotope masses, one 
might first consider two or more linear, two-parameter calibrations over more restricted mass ranges. Such adjustment of the calibration function should be required infrequently. In gamma-ray NDA at Los Alamos, the accuracy has more often been limited by inhomogeneity or of excessive particle size in the sample, and a single one-parameter linear calibration over the range required has nearly always been sufficient.

The extent to which one may safely extrapolate even a linear calibration beyond its data points will usually depend on whether the extrapolation is toward lower or highcr masses of the assay isotope than those contained in the standards. In gamma-ray NDA, the requirement to "span the range" is more burdensome and least necessary when the extrapolation is toward smaller assay masses than those contained in the standards. If the expected isotope masses in the unknowns include levels that give low count rates, which result in poor precision in a normal assay interval, then the time required to count a standard of equivalent concentration to a precision justifying its inclusion in calibration data rapidly becomes inconveniently long. At low concentrations of the assay isotope, the self-attenuation is usually dominated by the matrix, even for the high- $Z$ elements encountered in special nuclear materials, and so $C F(A T)$ usually changes very slowly over a wide range of low concentrations. Similarly, the count rates are usually low so that $\mathrm{CF}(\mathrm{RL})$ not only changes slowly but is small and accurately determined. As a result, one usually has high confidence in extrapolating from an assay isotope mass, which provides reasonable statistics in a reasonable time down to the lowest detectable levels. As an example, consider the assay of ${ }^{235} \mathrm{U}$ solutions by the 185.7-keV gamma ray. For reasonably sized samples $(>25 \mathrm{ml})$, a concentration of $\sim 10 \mathrm{~g} / \mathrm{l}^{235} \mathrm{U}$ may well give a precision of $\sim 0.5 \%$ in $\sim 1000 \mathrm{~s}$. A sample of $0.1 \mathrm{~g} / \mathrm{l}{ }^{235} \mathrm{U}$ concentration would have nearly the same $\mathrm{CF}(\mathrm{AT})$ and would give a precision of $\sim 5 \%$ in a $1000-\mathrm{s}$ assay, which might well be quite acceptable for the assay result at that concentration. However, it would take $\sim 100000$ s to count a $0.1 \mathrm{~g} / \mathrm{l}{ }^{235} \mathrm{U}$ standard to $0.5 \%$ precision, which one might well require if including it in the calibration data. A great deal of time can be and has been wasted in counting very low level standards.

The extrapolation to mass values higher than those in the standards must in general be approached more cautiously, especially if the highest mass standard is at a level where both $C F(A T)$ and $C F(R L)$ are changing rapidly or where the gross count rates are approaching the limits of the ability of the electronics to maintain adequate resolution and satisfactory full-energy peak shape. One should probably confirm the performance of the system at the higher masses and count rates with an approximately known source material of some sort even if no standard exists at the desired mass level. For example, if it has been confirmed that the system is able to accurately measure a transmission of $1 \%$ at a count rate of $50000 \mathrm{~s}^{-1}$ with overall satisfactory performance of the electronics, then one would have reasonable confidence in assaying an unknown with $1 \%$ transmission at $50000 \mathrm{~s}^{-1}$ even if the highest mass standard has an $\sim 2 \%$ transmission and gives a gross rate of $\sim 40000 \mathrm{~s}^{-1}$. If the entire expected range of assay isotope in the unknowns is such to give only modest count rates and small and slowly varying values of $C F(R L)$ and $\mathrm{CF}(\mathrm{AT})$, then one is safer in extrapolating upward over a greater fraction of the expected mass range.

A major point being emphasized here, and in the whole report, is that even though one will always want several standards to calibrate a gamma-ray NDA system and to confirm the accuracy of the calibration, that number can be much smaller than would be needed if the requirements of chemical and physical similarity and of "spanning the mass range" were strictly observed.

By way of final comment, the possession of an appropriate set of standards does not compensate for lack of knowledge of how to use them or for maladjusted or malfunctioning equipment, inappropriate assay geometries, incorrect expressions for the correction factors, or assay samples that do not adequately meet the requirements on uniformity and homogeneity. All the items are important factors in achieving accurate gamma-ray assays, and none can be safely neglected. When all the pertinent factors are properly addressed, including proper and efficient use of calibration standards, which is the subject of this report, gamma-ray NDA can provide economical, timely, and accurate assays for many classes of gamma-ray-emitting materials.

\section{REFERENCES}

1. American National Standard Guide to Calibrating Nondestructive Assay Systems, ANSI N15.20-1975 (American National Standards Institute, 1975).

2. R. H. Augustson and T. D. Reilly, "Fundamentals of Passive Nondestructive Assay of Fissionable Material," Los Alamos Scientific Laboratory report LA-5651-M (September 1974).

3. T. D. Reilly and J. L. Parker, "A Guide to GammaRay Assay for Nuclear Material Accountability," Los Alamos Scientific Laboratory report LA-5794M (March 1975).

4. Glenn F. Knoll, Radiation Detection and Measurement (John Wiley \& Sons, New York, 1979). 
5. F. Adams and R. Dams, Applied Gamma-Ray Spectrometry (Pergamon Press, Inc., New York, 1970).

6. G. P. Westphal, "Real-Time Correction of Counting Losses in Nuclear Pulse Spectroscopy," $J$. Radioanal. 70 (2), 387-391 (1982).

7. R. D. Evans, The Atomic Nucleus (McGraw-Hill, Inc., New York, 1955), Chaps. 23-25.

8. J. -P. Francois, "On the Calculation of the SelfAbsorption in Spherical Radioactive Sources," Nucl. Instrum. Methods 117, 153-156 (1974).

9. J. L. Parker, “A Correction for Gamma-Ray SelfAttenuation in Regular Heterogeneous Materials," Los Alamos National Laboratory report LA-8987MS (September 1981).

10. J. L. Parker and T. D. Reilly, "Bulk Sample SelfAttenuation Correction by Transmission Measurement," Proc. ERDA X- and Gamma-Ray Symposium, Ann Arbor, Michigan, May 19-21, 1976 (Conf. 760539).

11. J. H. Hubbell, "Photon Cross Sections, Attenuation Coefficients, and Energy Absorption Coefficients from $10 \mathrm{keV}$ to $100 \mathrm{GeV}$," National Bureau of Standards report NSRDS-NBS 29 (1969).
12. W. H. McMaster, N. Kerr Del Grande, J. H. Mallett, and J. H. Hubbell, "Compilation of X-Ray Cross Sections," Lawrence Radiation Laboratory report UCRL-50174, Sec. II, Rev. 1 (1969).

13. J. E. Cline, "A Technique of Gamma-Ray Detector Absolute Efficiency Calibration for Extended Sources," Proc. American Nuclear Society Topical Conference on Computers in Activation Analysis and Gamma Ray Spectroscopy, Mayaguez, Puerto Rico, (1978), pp. 185-196 (Conf. 780421).

14. "Self-Shielding Correction for Photon Irradiation of Slab and Cylindrical Samples," Gulf General Atomic, Inc., Progress Report GA-9614 (July 1, 1968-June 30, 1969).

15. Handbook of Mathematical Functions With Formulas, Graphs, and Mathematical Tables, Milton Abramowitz and Irene A. Stegun, Eds., National Bureau of Standards, Applied Mathematics Series 55 (1970).

16. Yardley Beers, Introduction to the Theory of Error (Addison-Wesley Publishing Co., Inc., Reading, Massachusetts, 1962).

17. Philip R. Bevington, Data Reduction and Error Analysis for the Physical Sciences (McGraw-Hill Book Company, New York, 1969). 